Nuclear Physics B292 (1987) 813-852

North-Holland, Amsterdam

\title{
AN EVALUATION OF THE GRAVITON PROPAGATOR IN DE SITTER SPACE
}

\author{
Bruce ALLEN and Michael TURYN \\ Department of Physics and Astronomy, Tufts University, Medford, Ma 02155, USA
}

Received 9 February 1987

(Revised 14 April 1987)

\begin{abstract}
The graviton propagator in four-dimensional de Sitter space is found in closed form. The vacuum state is taken to be the de Sitter-invariant "euclidean" or "Gibbons-Hawking" vacuum. The gauge-fixing term used is the standard choice introduced by Christensen and Duff. The propagator is given explicitly in this gauge, and is found to be finite for points that are not null-related. The method used is new: mode-sums on the four-sphere are expressed as maximally symmetric bitensors. The result is then given in a completely geometric and coördinate-free form. This same method can easily be used for maximally symmetric spaces of general dimension, including anti-de Sitter space with supersymmetric boundary conditions, and for different choices of gauge.
\end{abstract}

\section{Introduction}

\subsection{DE SITTER SPACE}

De Sitter space interests different people for different reasons. It describes an otherwise empty spacetime in the presence of a constant, static energy density [1]. For some, it is of interest on purely pedagogical grounds: the spacetime is as symmetric as ordinary Minkowski space, but it also has a constant non-zero curvature. As such, it provides a tractable example illustrating the effects of curvature, most notably the Gibbons-Hawking radiation [2]. For others, it is of interest because the observed cosmological constant $\Lambda$ is so small. Since de Sitter space is essentially "Minkowski space in the presence of a non-zero $\Lambda$ ", there is hope that one might find therein some inconsistency whose only resolution forces $\Lambda$ to vanish [3].

Finally, for the majority of physicists, de Sitter space becomes of interest in the light of current understanding of gauge theories. Both simple and unified gauge theories suggest the presence of phase transitions in the early universe, and that the 
unbroken-symmetry phase has a "latent heat" which would act exactly like a non-zero $\Lambda$ [4]. It is further known (modulo certain technical details and difficulties) that such theories give rise to "inflationary" models of the universe, which are to date the most successful and predictive theories available about the origins of the universe. In these models, spacetime is not exactly de Sitter space, but does pass through a long period for which $\Lambda$ is non-zero; the space then approaches de Sitter space in an exponentially asymptotic fashion. Thus if we wish to understand (for example) the physics of elementary particles at energies not likely to be produced in the laboratory, we would do well to understand de Sitter space.

\subsection{THE PROPAGATOR}

The purpose of this paper is to present, for the first time, a formula for the graviton propagator in four-dimensional de Sitter space. In the linearised or semiclassical theory of gravity, which is believed to be a good approximation to a true theory of quantum gravity at energy scales lower than that of the Planck scale of $10^{19} \mathrm{GeV}$, the propagator for two spacetime points $x$ and $x^{\prime}$ gives the tree-level amplitude for a graviton to get from $x$ to $x^{\prime}$. For this reason it is also referred to as the two-point function.

Here, we calculate the "coordinate space" two-point function for spacelike separated points. The Feynman (time-ordered) correlation function can then be obtained trivially by analytic continuation. The other correlation functions - commutator, anti-commutator, advanced, and retarded - can then be easily found [5]. Because the propagator for spacelike points is found first, all our calculations can be done on a riemannian 4-sphere of radius $a$ such that $a^{2}=3 / \Lambda^{2}$. There, the metric signature is $(++++)$, and the two-point function so obtained is exactly the same as that for spacelike-separated points in the physical spacetime (with lorentzian signature $(-+++))[6]$.

In de Sitter space, there exists a one-parameter family of vacuum states which are invariant under the group of spacetime isometries [7]. However, within this family only one vacuum state has a two-point function with either of the following (desirable) properties:

(i) It has the Hadamard form for short distances.

(ii) It is nonsingular for two points that are not null-related.

We will find the two-point function for this vacuum state, which is often called the "euclidean" or "Gibbons-Hawking" vacuum. It is singled out in our calculation by virtue of the technique employed in finding the propagator: it is the sum over mode-functions regular on the euclidean section of de Sitter space (the 4-sphere). It remains to be verified that the corresponding Fock state exists, but this is almost certainly true, as the nonexistence of such a state generally means that the two-point function is either extremely pathological or simply fails to exist. 
The potential usefulness of the graviton propagator seems clear. First of all, there are unresolved technical questions about this two-point function in the existing literature [3]. The most outstanding of these concern the infrared or long-distance behavior of the two-point function. We will have more to say about this later, but our results clearly answer at least one of these questions: the two-point function has no intrinsic infrared divergence, although poor choices of gauge can give rise to them. Our choice of gauge, the "standard" gauge of Christensen and Duff [8], is free of any such divergence. Unlike the case of flat space, the absence of these divergences turns out to be unrelated to the behavior of the two-point function at large distances. In fact, although the propagator is free of infrared divergences, it still grows in an unbounded fashion for large spacelike separations. Another important feature of the gauge chosen is that it does not break de Sitter invariance.

Another interesting application of the propagator will present itself in the examination of the behavior of idealised gravitational wave (i.e., graviton particle) detectors in de Sitter space. This can be done by generalising the work of Unruh [9] and others to the spin-two case. For the inflationary universe, the two-point function can thus be used to study the spectrum of the gravitational-wave noise background. In inflationary models this backround is believed to arise entirely from the zero-point fluctuations (equivalently, the Gibbons-Hawking radiation) during the de Sitter epoch. Thus our results can be applied to a systematic study of the microwave radiation induced (via the Sachs-Wolfe effect [10]) from the backround spectrum of gravity-wave noise.

This work will also permit a critical examination of some of the ideas which can be loosely described as "the decay of the cosmological constant" [11]. One way to do this is via a study of the unitarity of certain scattering amplitudes. This can most easily be done using dimensional regularisation, and it is for this reason that our paper will shortly be followed by a similar one generalising our results to a de Sitter space of arbitrary dimension.

Our paper contains one new technique. Typically, there are two ways to find correlation functions: mode-sums, and position space methods; in flat space, these two methods correspond to the complementary momentum- and coördinate-space representations. In our calculation, we were forced to use both methods together, and were thus led to the remarkably simple connection between them. In the mode-sum method, the two-point function is realised as an infinite sum over a set of irreducible representations of $\mathrm{SO}(5)$, which are labelled by the d'alembertian ( $\square$ ). De Sitter invariance forces all degenerate representations labelled by a given eigenvalue of $\square$ to appear with equal weight in the mode-sum. In the coördinate-space method, de Sitter invariance implies that the two-point function is a maximally symmetric bitensor [6]. A maximally symmetric bitensor is a geometrical object carrying spacetime indices, which depends on one-dimensional functions of the geodesic distance $\mu\left(x, x^{\prime}\right)$ between $x$ and $x^{\prime}$. What we found permits one to express each term in the mode-sum (which is sum over all the degenerate modes for a given 
eigenvalue of $\square$ ) as a maximally symmetric bitensor - roughly speaking, as a function of geodesic distance alone. A complete and systematic development of this correspondence would require yet another paper, and will not be attempted here. The reader interested in such technical matters may note, however, that the expression of a single term in the mode-sum as a bitensor allows us to sidestep certain obstacles that would otherwise present formidable impasses in our calculation. This is the case whenever previously-obtained objects, such as the scalar propagator for de Sitter space, must be modified by the removal of particular terms in their mode-sum expressions. As a final technical point, note that all of our calculations are done in a completely coördinate-free manner. Similarly, we make extensive use of scalar, vector, and tensor eigenfunctions of the d'alembertian operator $\square$, but not of their forms in any particular coördinate system. Only general formal properties of these eigenfunctions are needed for the calculation.

\subsection{STRUCTURE}

This paper is organised as follows. In sect. 2 we derive expressions for the graviton propagator, both as a formal infinite sum over tensor modes on the 4-sphere, and as a maximally symmetric bitensor depending on five undetermined functions of the geodesic distance $\mu$. The propagator can be naturally represented as a sum of four terms. We call them the TT (transverse-traceless), T (transverse-vector), L (longitudinal scalar), and PT (pure-trace scalar) terms. In sect. 3, we find the PT part to be simply related to a standard result for the propagator of a scalar field. In sect. 4, we find the longitudinal part of the propagator. In this case, one can use a partial fraction decomposition of the mode-sum to relate the longitudinal piece of the propagator to the same standard scalar-field result. In sect. 5, we find the transverse part of the propagator by exploiting the relationship between the modesum obtained in sect. 2, and a recent result of Allen and Jacobson for the massive vector propagator expressed as a maximally symmetric bitensor [6]. In sect. 6, we find the transverse-traceless part of the propagator. It is in a certain sense the only fundamentally new object found herein, since it cannot be related to the spin- 0 and spin-1 propagators already available. This calculation is complicated by the inhomogeneous equation of motion obeyed by the transverse-traceless term, which is in fact analogous to the case of the propagator for a massless vector field in de Sitter space [6]. Finally, in sect. 7 we add together the terms that we have found to obtain the full graviton propagator. In this section, we also examine the flat space $(\Lambda \rightarrow 0)$, short distance $\left(x \rightarrow x^{\prime}\right)$, antipodal $(\mu \rightarrow \pi a)$, and large distance $(\mu \rightarrow \infty)$ limits, and discuss the analytic structure of the propagator. This is followed by a concluding section. There are two technical appendices. The first (appendix A) contains useful calculational results for maximally symmetric spacetimes, and explicit details of the eigenfunctions of $\square$ employed in the text. In the second (appendix B), the source-term for the inhomogeneous equation of motion obeyed by the transverse-traceless piece of the propagator is evaluated in terms of elementary functions. 


\section{The graviton propagator}

In this section, we define the graviton propagator on the 4-sphere, where a standard choice of gauge has been made for the second-order graviton action. Maximally symmetric bitensors are defined for the 4-sphere, and their relevance to the symmetric function is established. Finally, the propagator is found formally as the inversion of the action by making use of a set of tensor mode-functions on the sphere, and the relationship between the bitensors and the mode-sums is explored.

\subsection{THE ACTION AND THE PROPAGATOR}

We start with the standard gravitational action

$$
S=-\frac{1}{16 \pi \mathscr{G}} \int_{V}(R-2 \Lambda) \mathrm{d} V
$$

where $\mathscr{G}$ is Newton's constant (we work in units for which $\hbar=c=1$, but $\mathscr{G}$ is retained explicitly), $V$ the manifold over which the integration is performed, and $R$ the Ricci scalar for that manifold. $\Lambda$ is the cosmological constant of the Einstein equation obtained from the first-order variation of the action (2.1). We consider the second-order effect of a small perturbation $h_{a b}$ on this action for a maximally symmetric spacetime with background metric $g_{a b}$. A maximally symmetric spacetime is an $n$-dimensional manifold admitting the maximum number of global Killing fields; there is no preferred direction selected at any point [6]. Curvature identities for maximally symmetric spaces (for which see appendix A) allow us to write the second-order variation in the action (2.1) on a 4-sphere of radius $a$ as $[12,13]$

$$
\delta^{(2)} S+S_{\mathrm{GF}}=\frac{1}{2} \rho \int h_{a b} W^{a b c d} h_{c d} \mathrm{~d} V
$$

for

$$
W^{a b c d}=\frac{1}{2}\left(g^{a c} g^{b d}+g^{a d} g^{b c}\right)\left(-\square+\frac{2}{3} \Lambda\right)+\frac{1}{2} g^{a b} g^{c d}\left(\square+\frac{2}{3} \Lambda\right),
$$

with $\rho=(32 \pi \mathscr{G})^{-1}$, and $\Lambda=3 a^{-2}$. This action incorporates the gauge-fixing term

$$
S_{\mathrm{GF}}=\rho \int\left(\nabla_{e}\left(h^{e f}-\frac{1}{2} h_{k}^{k} g^{e f}\right)\right)^{2} \mathrm{~d} V
$$

A more general choice of gauge will be considered in a future paper, as well as a general number of dimensions. It is sufficient to note that this choice of gauge-fixing term actually fixes the gauge, corresponding to the choice $\varepsilon=\frac{1}{2}$ in the language of [14]. 
We are looking for the de Sitter space two-point function

$$
G^{a b c^{\prime} d^{\prime}}\left(x, x^{\prime}\right)=\left\langle 0\left|h^{a b}(x) h^{c^{\prime} d^{\prime}}\left(x^{\prime}\right)\right| 0\right\rangle,
$$

where $|0\rangle$ is the euclidean vacuum state [7]. Defined in this way, the two-point function can be represented as a maximally symmetric bitensor [6].

\subsection{MAXIMALLY SYMMETRIC BITENSORS}

A bitensor $T^{a \ldots b^{\prime}}{ }_{c \ldots d^{\prime} \ldots}\left(x, x^{\prime}\right)$ is any function of two points that transforms as a tensor under coördinate transformations at either point. It can have any number of primed or unprimed indices, associated with the two points. In general, a prime on an index indicates that the index "lives" in $x$ "s tangent space; unprimed indices live at $x$. When we do not need to explicitly show an object's spacetime indices, the object will appear in a boldface type (e.g. $T, x)$.

A maximally symmetric bitensor is a bitensor that remains invariant under all isometries of the manifold [6]. The expectation value (2.5) is an example of such an object, because the euclidean vacuum state is maximally symmetric, and the operator inside does not break de Sitter invariance. We can further restrict the set of possible maximally symmetric bitensors making up the propagator (2.5) by noting that they must possess the index symmetries

$$
a \rightleftharpoons b, \quad c^{\prime} \rightleftharpoons d^{\prime} \quad \text { and } \quad\left[(a b) \rightleftharpoons\left(c^{\prime} d^{\prime}\right) \& x \rightleftharpoons x^{\prime}\right] .
$$

For any maximally symmetric space, there are five distinct maximally symmetric objects possessing these index symmetries. Thus the propagator can be expressed as a sum of the form

$$
o^{1} O_{1}^{a b c^{\prime} d^{\prime}}+o^{2} O_{2}^{a b c^{\prime} d^{\prime}}+o^{3} O_{3}^{a b c^{\prime} d^{\prime}}+o^{4} O_{4}^{a b c^{\prime} d^{\prime}}+o^{5} O_{5}^{a b c^{\prime} d^{\prime}},
$$

where $\left\{O_{1}^{a b c^{\prime} d^{\prime}}, \ldots, O_{5}^{a b c^{\prime} d^{\prime}}\right\}$ is a basis set of five bitensors with the requisite symmetries, and $\left\{o^{1}(\mu), \ldots, o^{5}(\mu)\right\}$ are undetermined functions of the geodesic distance $\mu$ between $x$ and $x^{\star \star}$. When we wish to represent an object with the propagator's symmetries, we will use a capital letter for the object, and the equivalent small letter for its coëfficient functions. For example, we will say " $\boldsymbol{G}_{\mathrm{T}}$ is given by $g_{\mathrm{T}}^{1}=c^{1}, \ldots, g_{\mathrm{T}}^{5}=c^{5}$, meaning

$$
G_{\mathrm{T}}^{a b c^{\prime} d^{\prime}}=c^{1} O_{1}^{a b c^{\prime} d^{\prime}}+\cdots+c^{5} O_{5}^{a b c^{\prime} d^{\prime}} .
$$

Our five basis bitensors can be built-up from the metric and three simple objects [6], which are shown in fig. 1.

These objects are:

(i) the metric tensor, $g^{a b}$;

(ii) the unit tangents at $x$ and $x^{\prime}, n^{a}=\nabla^{a} \mu$ and $n^{a^{\prime}}=\nabla^{a^{\prime}} \mu$;

"In this paper, "a function of $\mu$ " should be taken to mean a function of $\mu\left(x, x^{\prime}\right)$ when a geodesic does in fact connect the points $x$ and $x^{\prime}$, or the analytic extension of that function otherwise $[6,27]$. 


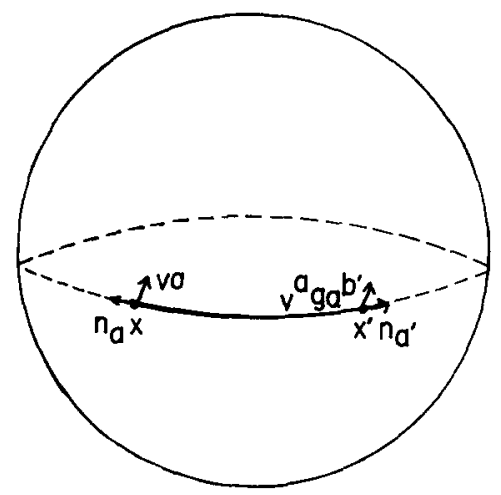

Fig. 1. The 4-sphere - a maximally symmetric space. Two points $x$ and $x^{\prime}$ in a maximally symmetric space determine preferred directions at those points. They are tangent vectors to the geodesics joining $x$ and $x^{\prime}$. The unit vectors $n_{a}$ at $x$ and $n_{a^{\prime}}$ at $x^{\prime}$ point away from the geodesic. The vector $V^{a} g_{a}{ }^{b^{\prime}}$ at $x^{\prime}$ is obtained by parallel transport of the vector $V^{a}$ along the geodesic from $x$ to $x^{\prime}$. Hence $n_{a}=-g_{a}{ }^{b^{\prime}} n_{b^{\prime}}$.

(iii) the parallel propagator $g^{a e^{\prime}}$, which has the following properties:

$$
\begin{aligned}
& g^{a e^{\prime}} n_{a}=-n^{e^{\prime}} \quad \text { and } \quad g^{a e^{\prime}} n_{e^{\prime}}=-n^{a} \\
& g^{a e^{\prime}} g_{e^{\prime} b}=g_{b}^{a}
\end{aligned}
$$

The basic rules for manipulation of these bitensors can be found in table 1 below, and other useful results can be found in appendix A. In this paper, we will denote the basis bitensors by $\left\{\boldsymbol{O}_{k}: k=1 \ldots 5\right\}$, defining them via

$$
\begin{aligned}
& O_{1}^{a b c^{\prime} d^{\prime}}=g^{a b} g^{c^{\prime} d^{\prime}}, \\
& O_{2}^{a b c^{\prime} d^{\prime}}=n^{a} n^{b} n^{c^{\prime} n^{d^{\prime}},} \\
& O_{3}^{a b c^{\prime} d^{\prime}}=g^{a c^{\prime}} g^{b d^{\prime}}+g^{a d^{\prime}} g^{b c^{\prime},} \\
& O_{4}^{a b c^{\prime} d^{\prime}}=g^{a b} n^{c^{\prime}} n^{d^{\prime}}+n^{a} n^{b} g^{c^{\prime} d^{\prime}}, \\
& O_{5}^{a b c^{\prime} d^{\prime}}=4 n^{(a} g^{b)\left(c^{\prime}\right.} n^{\left.d^{\prime}\right)} .
\end{aligned}
$$

These have been defined in order of increasing difficulty of manipulation. Note that $O_{4}^{a b c^{\prime} d^{\prime}}$ stands alone in that it cannot be reproduced by the separate symmetrisation of the primed and unprimed indices of a single bitensor term, as in the definition of 
TABLE 1

Bitensor manipulation rules

$$
\begin{gathered}
\nabla^{a} n^{b}=A\left(g^{a b}-n^{a} n^{b}\right) \\
\nabla^{a} n^{c^{\prime}}=C\left(g^{a c^{\prime}}+n^{a} n^{c^{\prime}}\right) \\
\nabla^{a} g^{b c^{\prime}}=-(A+C)\left(g^{a b} n^{c^{\prime}}+g^{a c^{\prime}} n^{b}\right)
\end{gathered}
$$

where

$$
\begin{gathered}
A=a^{-1} \cot (\mu / a) \\
C=-a^{-1} \csc (\mu / a)
\end{gathered}
$$

Note that

$$
\begin{aligned}
& C^{2}-A^{2}=a^{-2} \\
& \frac{\mathrm{d} A}{\mathrm{~d} \mu}=-C^{2} \\
& \frac{\mathrm{d} C}{\mathrm{~d} \mu}=-A C
\end{aligned}
$$

$O_{5}^{a b c^{\prime} d^{\prime}}$. This makes it convenient for us to define an auxillary object $\boldsymbol{O}_{6}$ via

$$
\dot{O}_{6}^{a b c^{\prime} d^{\prime}}=g^{a b} n^{c^{\prime}} n^{d^{\prime}}-n^{a} n^{b} g^{c^{\prime} d^{\prime}} .
$$

No object with our propagator's index symmetries can have a non-zero $\boldsymbol{O}_{6}$ term. Since this is so, we will not bother to give the $\boldsymbol{O}_{6}$ term for objects found in this paper - it can be assumed to be 0 unless otherwise noted.

\subsection{EQUATION OF MOTION AND MODE - SUM INVERSION}

The propagator $\boldsymbol{G}$ defined in (2.5) obeys the equation of motion $[5,15]$

$$
W^{a b c d} G_{c d}^{e^{\prime} f^{\prime}}\left(x, x^{\prime}\right)=1^{a b e^{\prime} f^{\prime}}\left(x, x^{\prime}\right) \equiv \frac{1}{2} O_{3}^{a b e^{\prime} f^{\prime}} \delta^{4}\left(x, x^{\prime}\right) .
$$

The factor of $\rho$ has been set equal to 1 , and will be restored in the final results. Using a set of tensor mode functions defined on the 4-sphere, (2.8) can be inverted, and the inverted expression evaluated using the vector and scalar propagators found in $[6,14]$. We will obtain the propagator as a sum of five tensor mode-sum pieces in this section, and spend the rest of this paper evaluating them.

We start with $\left\{\varphi_{n}^{i}\right\},\left\{\xi_{n}^{i a}\right\}$, and $\left\{h_{\mathrm{TT} n}^{i a b}\right\}$, the spin-0, spin-1, and spin-2 eigenfunctions of $\square$ on the 4-sphere. Details of their respective spectra and degeneracies can be found in $[14,19,21]$, and in appendix $A$ of this paper. Their eigenvalues will be 
denoted by

$$
\begin{gathered}
\square \varphi_{n}^{i}=\lambda_{n}^{(0)} \varphi_{n}^{i}, \\
\square \xi_{n}^{i a}=\lambda_{n}^{(1)} \xi_{n}^{i a}, \\
\square h_{\mathrm{T} T n}^{i a b}=\lambda_{n}^{(2)} h_{\mathrm{T} T n}^{i a b} .
\end{gathered}
$$

The modes are orthogonal in the following sense:

$$
\begin{aligned}
\int \varphi_{m}^{j}(x) \varphi_{n}^{i}(x) \mathrm{d} V & =\int \xi_{m}^{j a}(x) \xi_{n a}^{i}(x) \mathrm{d} V \\
& =\int h_{\mathrm{TT} m}^{j a b}(x) h_{\mathrm{TT} n a b}^{i}(x) \mathrm{d} V=\delta^{j i} \delta_{m n},
\end{aligned}
$$

where the integrals are over the 4-sphere (as are all integrals in this paper involving mode functions). We will usually drop the degeneracy index $i$ used in (2.9); all summations over the spectral index $(n=0,1,2,3 \ldots$ in the above) will then include an implicit sum over the degenerate modes.

Starting with the scalar and vector eigenfunctions, one can obtain tensor mode functions using the gradient operator and the backround metric tensor $g^{a b}$. They are defined by

$$
\begin{aligned}
& \chi_{n}^{a b}=\frac{1}{2} g^{a b} \varphi_{n}, \quad n=0,1,2,3, \ldots, \\
& W_{n}^{a b}=\frac{\left(\nabla^{a} \nabla^{b}-\frac{1}{4} g^{a b} \square\right) \varphi_{n}}{\sqrt{\lambda_{n}^{(0)}\left(\frac{3}{4} \lambda_{n}^{(0)}+\Lambda\right)}}, \quad n=2,3,4 \ldots, \\
& V_{n}^{a b}=\frac{\nabla^{(a} \xi_{n}^{b)}}{\sqrt{-\frac{1}{2}\left(\lambda_{n}^{(1)}+\Lambda\right)}}, \quad n=1,2,3 \ldots
\end{aligned}
$$

The $\chi$ 's will be referred to as the "pure-trace" tensor modes; the traceless $W$ and $V$ modes will be called the longitudinal and transverse tensor modes. These modes are orthonormal in the same sense as the $h_{\mathrm{TT} n}^{i a b}$ 's, that is

$$
\begin{aligned}
\int \chi_{m}^{j a b}(x) \chi_{n a b}^{i}(x) \mathrm{d} V & =\int W_{m}^{j a b}(x) W_{n a b}^{i}(x) \mathrm{d} V \\
& =\int V_{m}^{j a b}(x) V_{n a b}^{i}(x) \mathrm{d} V=\delta^{j i} \delta_{m n} .
\end{aligned}
$$

In addition, the four types of modes $\chi, W, V$ and $\boldsymbol{h}_{T T}$ are orthogonal to each other ${ }^{\star}$.

\footnotetext{
* Note that the lower bounds on $n$ for these modes are necessary - we could not have defined the $\boldsymbol{W}$
} and $V$ modes for all non-negative $n$ 's, since $W_{0}(x), W_{1}^{i}(x)$, and $V_{0}^{i}(x)$ are all identically zero. 
It will be necessary to calculate $\square \boldsymbol{V}$ and $\square \boldsymbol{W}$, as the eigenvalues of these modes are shifted away from those of the eigenfunctions from which they were constructed. They are

$$
\begin{gathered}
\square \boldsymbol{V}=\left(\lambda_{n}^{(1)}+\frac{5}{3} \Lambda\right) \boldsymbol{V}, \\
\square \boldsymbol{W}=\left(\lambda_{n}^{(0)}+\frac{8}{3} \Lambda\right) \boldsymbol{W} .
\end{gathered}
$$

The result of performing a functional integral over the metric perturbations [14] shows that the graviton propagator takes the following form:

$$
\begin{aligned}
G^{a b c^{\prime} d^{\prime}}\left(x, x^{\prime}\right)= & \sum_{n=0}^{\infty} a_{n}\left[h_{\mathrm{TT} n}^{a b}(x) h_{\mathrm{T} T n}^{c^{\prime} d^{\prime}}\left(x^{\prime}\right)\right]+\sum_{n=1}^{\infty} b_{n}\left[V_{n}^{a b}(x) V_{n}^{c^{\prime} d^{\prime}}\left(x^{\prime}\right)\right] \\
& +\sum_{n=2}^{\infty} c_{n}\left[W_{n}^{a b}(x) W_{n}^{c^{\prime} d^{\prime}}\left(x^{\prime}\right)\right]+\sum_{n=0}^{\infty} d_{n}\left[\chi_{n}^{a b}(x) \chi_{n}^{c^{\prime} d^{\prime}}\left(x^{\prime}\right)\right] \\
& +\sum_{n=2}^{\infty} e_{n}\left[\chi_{n}^{a b}(x) W_{n}^{c^{\prime} d^{\prime}}\left(x^{\prime}\right)+W_{n}^{a b}(x) \chi_{n}^{c^{\prime} d^{\prime}}\left(x^{\prime}\right)\right] .
\end{aligned}
$$

As our tensor mode functions form a complete set on the 4-sphere, one may write the $S^{4}$ tensor $\delta$-function as

$$
\begin{aligned}
1^{a b c^{\prime} d^{\prime}}\left(x, x^{\prime}\right)= & \sum_{n=0}^{\infty} h_{\mathrm{T} \mathrm{T} n}^{a b}(x) h_{\mathrm{T} \mathrm{T} n}^{c^{\prime} d^{\prime}}\left(x^{\prime}\right)+\sum_{n=1}^{\infty} V_{n}^{a b}(x) V_{n}^{c^{\prime} d^{\prime}}\left(x^{\prime}\right) \\
& +\sum_{n=2}^{\infty} W_{n}^{a b}(x) W_{n}^{c^{\prime} d^{\prime}}\left(x^{\prime}\right)+\sum_{n=0}^{\infty} \chi_{n}^{a b}(x) \chi_{n}^{c^{\prime} d^{\prime}}\left(x^{\prime}\right) .
\end{aligned}
$$

We substitute the eigenfunction forms for $G$ (2.14) and the tensor $\delta$-function (2.15) into the equation of motion (2.8), and contract with $\boldsymbol{h}_{\mathrm{TT} m}, \boldsymbol{V}_{m}, \boldsymbol{W}_{m}$, and $\boldsymbol{\chi}_{m}$ in succession, and then with their primed (') equivalents. Integration with respect to $\mathrm{d} V$ and $\mathrm{d} V^{\prime}$ gives

$$
\begin{aligned}
& \left(-\lambda_{n}^{(2)}+\frac{2}{3} \Lambda\right) a_{n}=1 \\
& \left(-\lambda_{n}^{(1)}-\Lambda\right) b_{n}=1 \\
& \left(-\lambda_{n}^{(0)}-2 \Lambda\right) c_{n}=1 \\
& \left(+\lambda_{n}^{(0)}+2 \Lambda\right) d_{n}=1 \\
& \left(+\lambda_{n}^{(0)}+\Lambda\right) e_{n}=\left(-\lambda_{n}^{(0)}+2 \Lambda\right) e_{n}=0 .
\end{aligned}
$$


We may therefore write the propagator as a sum of four terms

where

$$
\boldsymbol{G}=\boldsymbol{G}_{\mathrm{TT}}+\boldsymbol{G}_{\mathrm{T}}+\boldsymbol{G}_{\mathrm{L}}+\boldsymbol{G}_{\mathrm{PT}},
$$

$$
\begin{aligned}
& G_{\mathrm{TT}}^{a b c^{\prime} d^{\prime}}\left(x, x^{\prime}\right)=\sum_{n=0}^{\infty} \frac{h_{\mathrm{T} T n}^{a b}(x) h_{\mathrm{T} n}^{c^{\prime} d^{\prime}}\left(x^{\prime}\right)}{-\lambda_{n}^{(2)}+\frac{2}{3} \Lambda}, \\
& G_{\mathrm{T}}^{a b c^{\prime} d^{\prime}}\left(x, x^{\prime}\right)=\sum_{n=1}^{\infty} \frac{V_{n}^{a b}(x) V_{n}^{c^{\prime} d^{\prime}}\left(x^{\prime}\right)}{-\lambda_{n}^{(1)}-\Lambda}, \\
& G_{\mathrm{L}}^{a b c^{\prime} d^{\prime}}\left(x, x^{\prime}\right)=\sum_{n=2}^{\infty} \frac{W_{n}^{a b}(x) W_{n}^{c^{\prime} d^{\prime}}\left(x^{\prime}\right)}{-\lambda_{n}^{(0)}-2 \Lambda}, \\
& G_{\mathrm{PT}}^{a b c^{\prime} d^{\prime}}\left(x, x^{\prime}\right)=\sum_{n=0}^{\infty} \frac{\chi_{n}^{a b}(x) \chi_{n}^{c^{\prime} d^{\prime}}\left(x^{\prime}\right)}{+\lambda_{n}^{(0)}+2 \Lambda} .
\end{aligned}
$$

These terms are referred to in this paper as the transverse-traceless, transverse, longitudinal, and pure-trace parts of the propagator ${ }^{\star}$. They will be evaluated using a variety of techniques, most of which have been used previously in [14]. Note that since $e_{n}=0$ for all $n$, there are no "mixed" terms in the propagator. This is because our choice of gauge-fixing term "diagonalises" the propagator.

\section{The pure-trace propagator}

The pure-trace part of the graviton propagator, $\boldsymbol{G}_{\mathrm{PT}}$, obeys a simple equation of motion. The total propagator (2.21) can be written as

$$
\boldsymbol{G}=\boldsymbol{G}_{\text {Traceless }}+\boldsymbol{G}_{\mathrm{PT}},
$$

where $\boldsymbol{G}_{\text {Traceless }}$ is traceless on both its primed and unprimed indices, and

$$
G_{\mathrm{PT}}^{a b c^{\prime} d^{\prime}}=\frac{1}{4} f\left(x, x^{\prime}\right) g^{a b} g^{c^{\prime} d^{\prime}}
$$

Taking the trace of the equation of motion (2.8) on its primed and unprimed indices, one finds

$$
(\square+2 \Lambda) f\left(x, x^{\prime}\right)=\delta^{4}\left(x, x^{\prime}\right) .
$$

* Note that the "transverse" piece of the propagator is not transverse in curved spaces $\left(\nabla \cdot \boldsymbol{G}_{\mathrm{T}}=0+\right.$ curvature terms). This is because the "transverse" tensor modes are not transverse, but are derivatives of transverse vector modes. The $V_{n}$ modes do obey the equation $\nabla_{a} \nabla_{b} V_{n}^{a b}=0$. 
In [6], the scalar propagator $G\left(\mathrm{~m}^{2} ; x, x^{\prime}\right)$ obeying the equation of motion

$$
\left(-\square+m^{2}\right) G\left(m^{2} ; x, x^{\prime}\right)=\delta^{n}\left(x, x^{\prime}\right)
$$

on an $n$-sphere of radius $a$ was found. The scalar propagator for four-dimensional de Sitter space is given by

$$
G\left(m^{2} ; x, x^{\prime}\right)=G\left(m^{2}, z\left(x, x^{\prime}\right)\right)=\frac{\Gamma\left(a_{0}\right) \Gamma\left(b_{0}\right)}{16 \pi^{2} a^{2}} F\left(a_{0}, b_{0} ; 2 ; z\right),
$$

where

$$
\begin{aligned}
& a_{0}=\frac{3}{2}+\sqrt{\frac{9}{4}-a^{2} m^{2}}, \\
& b_{0}=\frac{3}{2}-\sqrt{\frac{9}{4}-a^{2} m^{2}}, \\
& z=z\left(x, x^{\prime}\right)=\cos ^{2}(\mu / 2 a) .
\end{aligned}
$$

Here, $F(a, b ; c ; z)$ is ${ }_{2} F_{1}(a, b ; c ; z)$, the usual hypergeometric function. Comparing (3.2) and (3.3), one immediately finds that

$$
f\left(x, x^{\prime}\right)=-G\left(-2 \Lambda, z\left(x, x^{\prime}\right)\right) .
$$

One can therefore express the pure-trace piece of the propagator, using (3.1b), as

$$
\boldsymbol{G}_{\mathrm{PT}}=-\frac{1}{4} G(-2 \Lambda, z) \boldsymbol{O}_{1} .
$$

This can also be expressed as

$$
\begin{aligned}
& g_{\mathrm{PT}}^{1}=-\frac{1}{4} G(-2 \Lambda, z), \\
& g_{\mathrm{PT}}^{2}=g_{\mathrm{PT}}^{3}=g_{\mathrm{PT}}^{4}=g_{\mathrm{PT}}^{5}=0 .
\end{aligned}
$$

The function $G(-2 \Lambda, z)$ is finite for $z \neq 1$, but cannot be expressed in terms of elementary functions, although we will employ various approximate forms for it later on. Note that (3.7) is valid for de Sitter, flat, and anti-de Sitter spacetimes, if the appropriate form of the scalar propagator is used in each case. However, the form for $G(-2 \Lambda, z)$ used in this paper is that of de Sitter space.

\section{The longitudinal part of the propagator}

In sect. 2, the propagator is expressed as a sum of four terms. In this section, the mode-sum expression for the "longitudinal" part of the propagator, $\boldsymbol{G}_{\mathrm{L}}$, is evaluated 
by relating it to the propagator (3.4). This evaluation is new, although expression for $G_{L}$ as a function of the scalar propagator was obtained in [14], where it was shown to be finite for our choice of gauge.

One may evaluate (2.24) by using the definitions of the longitudinal modes $\boldsymbol{W}_{n}$ in terms of the scalar eigenfunctions (2.11b). One finds

$$
G_{L}^{a b c^{\prime} d^{\prime}}=\overline{\nabla^{a} \nabla^{b}} \overline{\nabla^{c^{\prime}} \nabla^{d^{\prime}} l} l\left(x, x^{\prime}\right),
$$

where the operator $\overline{\nabla^{a} \nabla^{b}}$ is the traceless part of $\nabla^{a} \nabla^{b}$,

$$
\begin{gathered}
\overline{\nabla^{a} \nabla^{b}}=\left(\nabla^{a} \nabla^{b}-\frac{1}{4} g^{a b} \square\right), \\
l\left(x, x^{\prime}\right)=\sum_{n=2}^{\infty} \frac{\varphi_{n}(x) \varphi_{n}\left(x^{\prime}\right)}{-\lambda_{n}^{(0)}\left(\lambda_{n}^{(0)}+2 \Lambda\right)\left(\frac{3}{4} \lambda_{n}^{(0)}+\Lambda\right)} .
\end{gathered}
$$

When a partial fraction decomposition of (4.2) is employed, one finds

$$
l\left(x, x^{\prime}\right)=\frac{1}{2 \Lambda^{2}} \sum_{n=2}^{\infty} \varphi_{n}(x) \varphi_{n}\left(x^{\prime}\right)\left[-\frac{1}{\lambda_{n}^{(0)}}-\frac{2}{\lambda_{n}^{(0)}+2 \Lambda}+\frac{3}{\lambda_{n}^{(0)}+\frac{4}{3} \Lambda}\right] .
$$

This form is a linear combination of three modified scalar propagators for three different mass values, because the scalar propagator in de Sitter space is

$$
G\left(m^{2}, z\left(x, x^{\prime}\right)\right)=\sum_{n=0}^{\infty} \frac{\varphi_{n}(x) \varphi_{n}\left(x^{\prime}\right)}{-\lambda_{n}^{(0)}+m^{2}} .
$$

Defining a modified propagator, where we drop the first $k$ modes, as

$$
\tilde{G}\left(k ; m^{2}, z\right) \equiv \sum_{n=k}^{\infty} \frac{\varphi_{n}(x) \varphi_{n}\left(x^{\prime}\right)}{-\lambda_{n}^{(0)}+m^{2}},
$$

one can re-write $l\left(x, x^{\prime}\right)$ as

$$
l\left(x, x^{\prime}\right)=\frac{1}{2 \Lambda^{2}}\left[\tilde{G}(2 ; 0, z)-3 \tilde{G}\left(2 ;-\frac{4}{3} \Lambda, z\right)+2 \tilde{G}(2 ;-2 \Lambda, z)\right]
$$

In appendix $\mathrm{A}$, we evaluate the first two terms in the scalar propagator mode-sum and show that

$$
\tilde{G}\left(2 ; m^{2}, z\right)=G\left(m^{2}, z\right)-\frac{3}{8 \pi^{2} a^{4} m^{2}}-\frac{15(2 z-1)}{8 \pi^{2} a^{2}\left(4+a^{2} m^{2}\right)} .
$$


In spite of appearances, the function $\tilde{G}\left(2, m^{2}, z\right)$ is finite at $m^{2}=0$ and at $m^{2}=$ $-\frac{4}{3} \Lambda$. The simple poles on the right-hand side of (4.7) are cancelled by corresponding poles in $G\left(\mathrm{~m}^{2}, z\right)$. One can evaluate the modified scalar propagator in (4.7) at $m^{2}=0$ and $m^{2}=-\frac{4}{3} \Lambda^{\star}$, obtaining

$$
\begin{gathered}
\tilde{G}(2 ; 0, z)=\frac{1}{32 \pi^{2} a^{2}}\left[\frac{2}{1-z}-4 \log (1-z)+\frac{17}{3}-30 z\right], \\
\tilde{G}\left(2 ;-\frac{4}{3} \Lambda, z\right)=\frac{1}{32 \pi^{2} a^{2}}\left[12(1-2 z) \log (1-z)+\frac{2}{1-z}+\frac{1}{5}(67-224 z)\right] .
\end{gathered}
$$

The function $\tilde{G}(2 ;-2 \Lambda, z)$ cannot be expressed in terms of elementary functions. This makes it convenient to split $G_{L}$ into a sum of two terms

$$
G_{\mathrm{L}}=G_{l_{1}}+G_{l_{2}}
$$

where

$$
\begin{aligned}
& G_{l_{1}}=\overline{\nabla \nabla} \overline{\nabla^{\prime} \nabla^{\prime}} \frac{1}{2 \Lambda^{2}}\left[\tilde{G}(2 ; 0, z)-3 \tilde{G}\left(2 ;-\frac{4}{3} \Lambda, z\right)\right], \\
& G_{l_{2}}=\overline{\nabla \nabla} \overline{\nabla^{\prime} \nabla^{\prime}} \frac{1}{\Lambda^{2}} \tilde{G}(2 ;-2 \Lambda, z) .
\end{aligned}
$$

Evaluating $G_{l_{1}}$ explicitly, we find

$$
\begin{aligned}
& g_{l_{1}}^{1}=\kappa\left[\frac{-6}{z-1}+\frac{2}{(z-1)^{2}}+\frac{1}{(z-1)^{3}}\right], \\
& g_{l_{1}}^{2}=\kappa\left[\frac{16}{z-1}-\frac{48}{(z-1)^{2}}+\frac{48}{(z-1)^{3}}\right], \\
& g_{l_{1}}^{3}=\kappa\left[\frac{9}{z-1}-\frac{4}{(z-1)^{2}}+\frac{1}{(z-1)^{3}}\right], \\
& g_{l_{1}}^{4}=\kappa\left[\frac{6}{z-1}-\frac{6}{(z-1)^{3}}\right], \\
& g_{l_{1}}^{5}=\kappa\left[\frac{10}{z-1}-\frac{12}{(z-1)^{2}}+\frac{6}{(z-1)^{3}}\right],
\end{aligned}
$$

* We evaluate (4.7) by taking the limit as $m^{2}$ approaches the poles at $m^{2}=0$ and $m^{2}=-\frac{4}{3} \Lambda$, representing the hypergeometric functions as power series in $z$ [16]. The resulting series may then be evaluated. The results obtained agree with the more general result obtained using formal properties of the scalar eigenfunctions (appendix A). 
where $\kappa=\left(1 / 288 \pi^{2} a^{2}\right)$. Note that this solution is regular as $z \rightarrow 0$ because we used the de Sitter space form of the scalar propagator.

Evaluation of $G_{l_{2}}$ is made easier by the result

$$
\overline{\nabla^{a} \nabla^{b}} \phi(z)=z(1-z) \frac{\mathrm{d}^{2} \phi}{\mathrm{d} z^{2}}\left[n^{a} n^{b}-\frac{1}{4} g^{a b}\right],
$$

for $\phi$ any biscalar function of $z$. This means in particular that

$$
\overline{\nabla \nabla} \overline{\nabla^{\prime} \nabla^{\prime}} \tilde{G}\left(2 ; m^{2}, z\right)=\overline{\nabla \nabla \nabla^{\prime} \nabla^{\prime}} \tilde{G}\left(0 ; m^{2}, z\right) \text {. }
$$

Thus it is not strictly necessary to introduce $\tilde{G}\left(2 ; m^{2}, z\right)$ in the first place, as it differs from the scalar propagator $G\left(m^{2}, z\right)$ by terms at most linear in $z$. Nevertheless, the fact that $\tilde{G}(2 ; 0, z)$ and $\tilde{G}\left(2 ;-\frac{4}{3} \Lambda, z\right)$ are finite - as opposed to the equivalent scalar propagators, which are infinite - makes their use desirable. On the other hand, there is no good reason to use $\tilde{G}(2 ;-2 \Lambda, z)$ instead of $G(-2 \Lambda, z)$, which is a finite scalar propagator. Using its equation of motion,

$$
\begin{aligned}
& (-\square-2 \Lambda) G(-2 \Lambda, z) \\
& \quad=-\frac{1}{a^{2}}\left[z(1-z) \frac{\mathrm{d}^{2}}{\mathrm{~d} z^{2}}+2(1-2 z) \frac{\mathrm{d}}{\mathrm{d} z}+2 \Lambda a^{2}\right] G(-2 \Lambda, z)=0,
\end{aligned}
$$

one can express second- and higher-order derivatives of $G(-2 \Lambda, z)$ with respect to $z$ in terms of the propagator and its first derivative. Defining $G^{-2 \Lambda} \equiv G(-2 \Lambda, z)$, we then obtain

$$
\begin{aligned}
& g_{l_{2}}^{1}=-\frac{4(2 z-1) G_{, z}^{-2 \Lambda}-11 G^{-2 \Lambda}}{12}-\frac{G_{, z}^{-2 \Lambda}+3 G^{-2 \Lambda}}{18 z}-\frac{G_{, z}^{-2 \Lambda}-3 G^{-2 \Lambda}}{18(z-1)}, \\
& g_{l_{2}}^{2}=-\frac{4(24 z-1) G_{, z}^{-2 \Lambda}-132 G^{-2 \Lambda}}{9}-\frac{8 G_{, z}^{-2 \Lambda}-24 G^{-2 \Lambda}}{3(z-1)}-\frac{4 G_{, z}^{-2 \Lambda}+12 G^{-2 \Lambda}}{9 z}, \\
& g_{l_{2}}^{3}=-\frac{G_{, z}^{-2 \Lambda}+3 G^{-2 \Lambda}}{18 z}-\frac{G_{, z}^{-2 \Lambda}-3 G^{-2 \Lambda}}{18(z-1)}, \\
& g_{l_{2}}^{4}=\frac{4(2 z-1) G_{, z}^{-2 \Lambda}-11 G^{-2 \Lambda}}{3}+\frac{G_{, z}^{-2 \Lambda}+3 G^{-2 \Lambda}}{3 z}+\frac{G_{, z}^{-2 \Lambda}-3 G^{-2 \Lambda}}{3(z-1)} \\
& g_{l_{2}}^{5}=\frac{2 G_{, z}^{-2 \Lambda}+6 G^{-2 \Lambda}}{9 z}-\frac{G_{, z}^{-2 \Lambda}-3 G^{-2 \Lambda}}{3(z-1)}-\frac{11 G_{, z}^{-2 \Lambda}}{9}
\end{aligned}
$$


These component coëfficient functions are regular as $z \rightarrow 0$.

One cannot evaluate (4.14) in terms of elementary functions ${ }^{\star}$, however limiting expressions for $G(-2 \Lambda, z)$ as $z \rightarrow 0, z \rightarrow 1$ and $z \rightarrow-\infty$ will later be presented. We note in passing that $G_{l_{2}}$ dominates the behavior of traceless part of the propagator in the limit $z \rightarrow-\infty$.

\section{The transverse part of the propagator}

In this section the transverse part of the spin-two propagator will be obtained from the scalar and vector propagators. To do this, the vector propagator is expressed as a mode-sum similar to those already encountered in the scalar case.

\subsection{THE TRANSVERSE PART AS A MODE-SUM}

We evaluate the mode-sum expression for $\boldsymbol{G}_{\mathrm{T}}$, which was obtained in sect. 2 (2.23):

$$
G_{\mathrm{T}}^{a b c^{\prime} d^{\prime}}=\sum_{n=1}^{\infty} \frac{V_{n}^{a b}(x) V_{n}^{c^{\prime} d^{\prime}}\left(x^{\prime}\right)}{-\left(\lambda_{n}^{(1)}+\Lambda\right)}
$$

Substituting in the definitions of the transverse mode-functions $V_{n}$ in terms of the vector mode-functions $\xi_{n}$ and their eigenvalues, one finds

$$
G_{\mathrm{T}}^{a b c^{\prime} d^{\prime}}=2 \sum_{n=1}^{\infty} \frac{\nabla^{(b} \xi_{n}^{a)} \nabla^{\left(d^{\prime}\right.} \xi_{n}^{\left.c^{\prime}\right)}}{\left(\lambda_{n}^{(1)}+\Lambda\right)^{2}}
$$

To evaluate (5.2), one must consider a mode-sum of the form

$$
\tilde{Q}^{a c^{\prime}}\left(k ; m^{2}, z\right)=\sum_{n=k}^{\infty} \frac{\xi_{n}^{a}(x) \xi_{n}^{c^{\prime}}\left(x^{\prime}\right)}{-\lambda_{n}^{(1)}+m^{2}+\Lambda}
$$

One can then take the partial derivative of (2.3) with respect to $m^{2}$ to define

$$
\bar{Q}^{a c^{\prime}}\left(k ; m^{2}, z\right)=-\frac{\partial}{\partial\left(m^{2}\right)} \tilde{Q}^{a c^{\prime}}\left(k ; m^{2}, z\right)=\sum_{n=k}^{\infty} \frac{\xi_{n}^{a}(x) \xi_{n}^{c^{\prime}}\left(x^{\prime}\right)}{\left(-\lambda_{n}^{(1)}+m^{2}+\Lambda\right)^{2}}
$$

One therefore obtains

$$
G_{\mathrm{T}}^{a b c^{\prime} d^{\prime}}=2 \nabla^{\left(d^{\prime}\right.} \nabla^{(b} \bar{Q}^{\left.a) c^{\prime}\right)}(1 ;-2 \Lambda, z)
$$

* One may evaluate the derivative of $G(-2 \Lambda, z)$ with respect to $z$ using an expression which shifts the parameters of the hypergeometric function, but this is of no use in simplifying the expressions. 
One can evaluate (5.5) using the vector and scalar propagators found in [6]. To do this, we will first need to find an expression for the vector propagator as a mode-sum over eigenfunctions of $\square$.

\subsection{THE VECTOR PROPAGATOR AS A MODE-SUM}

One can evaluate $\overline{\boldsymbol{Q}}$ by relating it to the vector and scalar propagators of [6]. The vector propagator $\boldsymbol{G}_{\text {spin-1 }}$ obeys the equation of motion

$$
\left(-\square g^{a b}+\nabla^{a} \nabla^{b}+m^{2} g^{a b}\right) G_{b c^{\prime}}^{\mathrm{spin}-1}=\delta^{4}\left(x, x^{\prime}\right) g_{{ }^{\prime}}^{a} .
$$

To express $\boldsymbol{G}_{\text {spin-1 }}$ as a mode-sum, one must introduce orthonormal (in the sense of sect. 2) spin-1 mode-functions $\left\{\left(1 / \sqrt{-\lambda_{n}^{(0)}}\right) \nabla^{a} \varphi_{n}\right\}$. These longitudinal modes are orthogonal to the familiar, transverse $\left\{\xi_{n}\right\}$ 's. Since the two sets together span the space of vectors on the $n$-sphere, we can write the spin-1 delta-function appearing in (5.6) as

$$
\delta^{4}\left(x, x^{\prime}\right) g^{a c^{\prime}}=\sum_{n=0}^{\infty} \xi_{n}^{a}(x) \xi_{n}^{c^{\prime}}\left(x^{\prime}\right)+\sum_{n=1}^{\infty} \frac{\nabla^{a} \varphi_{n}(x) \nabla^{c^{\prime}} \varphi_{n}\left(x^{\prime}\right)}{-\lambda_{n}^{(0)}} .
$$

One can then assume a mode-sum form for $\boldsymbol{G}_{\text {spin-1 }}$ :

$$
G_{\mathrm{spin}-1}^{a c^{\prime}}\left(m^{2}, z\right)=\sum_{n=0}^{\infty} a_{n} \xi_{n}^{a}(x) \xi_{n}^{c^{\prime}}\left(x^{\prime}\right)+\sum_{n=1}^{\infty} b_{n} \frac{\nabla^{a} \varphi_{n}(x) \nabla^{c^{\prime}} \varphi_{n}\left(x^{\prime}\right)}{-\lambda_{n}^{(0)}}
$$

and invert (5.6), obtaining

$$
G_{\text {spin-1 }}^{a c^{\prime}}\left(m^{2} ; x, x^{\prime}\right)=\sum_{n=0}^{\infty} \frac{\xi_{n}^{a}(x) \xi_{n}^{c^{\prime}}\left(x^{\prime}\right)}{-\lambda_{n}^{(1)}+\Lambda+m^{2}}+\left(\frac{1}{m^{2}}\right) \nabla^{a} \nabla^{c^{\prime}} \sum_{n=1}^{\infty} \frac{\varphi_{n}(x) \varphi_{n}\left(x^{\prime}\right)}{-\lambda_{n}^{(0)}}
$$

Note that for $m^{2}=0$, the vector propagator's equation of motion cannot be successfully inverted because the $b_{n}\left(\mathrm{~m}^{2}\right)$ of (5.8) are not determined. This is a characteristic of the massless spin- 1 action in the absence of an additional gauge-fixing term [5].

Expression (5.9) will be a bridge between (5.2), which gives the transverse propagator as a mode-sum, and the expression for the vector propagator given in ref. [6].

5.3. THE TRANSVERSE PROPAGATOR IN TERMS OF MODIFIED SCALAR AND VECTOR PROPAGATORS

Using $\tilde{\boldsymbol{Q}}$ as defined above, and the modified scalar propagator $\tilde{G}$ defined in (4.5), we can rewrite our expression for the vector two-point function (5.9) as

$$
G_{\mathrm{spin}-1}^{a c^{\prime}}\left(m^{2}, z\right)=\tilde{Q}^{a c^{\prime}}\left(0 ; m^{2}, z\right)+\frac{1}{m^{2}} \nabla^{a} \nabla^{c^{\prime}} \tilde{G}(1 ; 0, z),
$$


or

$$
\tilde{Q}^{a c^{\prime}}\left(0 ; m^{2}, z\right)=G_{\mathrm{spin}-1}^{a c^{\prime}}\left(m^{2}, z\right)-\frac{1}{m^{2}} \nabla^{a} \nabla^{c^{\prime}} \tilde{G}(1 ; 0, z)
$$

By adding $\tilde{Q}^{a c^{\prime}}\left(1 ; m^{2}, z\right)-\tilde{Q}^{a c^{\prime}}\left(0 ; m^{2}, z\right)$ to both sides of $(5.10 \mathrm{~b})$, we obtain

$$
\begin{aligned}
\tilde{Q}^{a c^{\prime}}\left(1 ; m^{2}, z\right)= & G_{\mathrm{spin}-1}^{a c^{\prime}}\left(m^{2}, z\right)-Q_{0}^{a c^{\prime}}\left(m^{2}, z\right) \\
& -\frac{1}{m^{2}} \nabla^{a} \nabla^{c^{\prime}} \tilde{G}(1 ; 0, z),
\end{aligned}
$$

where

$$
Q_{0}^{a c^{\prime}}\left(m^{2}, z\right) \equiv \tilde{Q}^{a c^{\prime}}\left(0 ; m^{2}, z\right)-\tilde{Q}^{a c^{\prime}}\left(1 ; m^{2}, z\right)=\sum_{i=1}^{10} \frac{\xi_{0}^{i a}(x) \xi_{0}^{i c^{\prime}}\left(x^{\prime}\right)}{2 \Lambda+m^{2}}
$$

One then takes the partial derivative of $(5.11)$ with respect to $\left(-m^{2}\right)$ to obtain

$$
\bar{Q}^{a c^{\prime}}\left(1 ; m^{2}, z\right)=\bar{G}_{\mathrm{spin}-1}^{a c^{\prime}}\left(m^{2}, z\right)-\frac{1}{m^{4}} \nabla^{a} \nabla^{c^{\prime}} \tilde{G}(1 ; 0, z)
$$

where

$$
\bar{G}_{\mathrm{spin}-1}^{a c^{\prime}}\left(m^{2}, z\right)=-\frac{\partial}{\partial\left(m^{2}\right)}\left(G_{\mathrm{spin}-1}^{a c^{\prime}}\left(m^{2}, z\right)-Q_{0}^{a c^{\prime}}\left(m^{2}, z\right)\right)
$$

We now have an expression for the transverse piece of the propagator in terms of the "modified propagators," $\bar{G}_{\text {spin-1 }}$ and $\tilde{G}$.

\subsection{EVALUATION OF THE MODIFIED PROPAGATORS}

To find $\bar{Q}^{a c^{\prime}}(1 ;-2 \Lambda, z)$ as given in $(5.13)$, one must evaluate $\tilde{G}(1 ; 0, z)$ and $\bar{G}_{\text {spin-1 }}^{a c^{\prime}}(-2 \Lambda, z)$. One can easily evaluate $\tilde{G}(1 ; 0, z)$, as $\tilde{G}(2 ; 0, z)$ and the additional term needed are given in sect. 4 and appendix $B$, obtaining

$$
\tilde{G}(1 ; 0, z)=\frac{1}{32 \pi^{2} a^{2}}\left[\frac{2}{1-z}-4 \log (1-z)-\frac{28}{3}\right] .
$$

In [6], an expression for the de Sitter space vector propagator $G_{\mathrm{spin}-1}^{a c^{\prime}}\left(m^{2}, z\right)$ is found. It is

$$
G_{\text {spin-1 }}^{a c^{\prime}}\left(m^{2}, z\right)=\alpha(\gamma) g^{a c^{\prime}}+\beta(\gamma) n^{a} n^{c^{\prime}}
$$


where the coëfficient functions are given in terms of a pair of differential operators:

$$
\begin{aligned}
& \alpha(\gamma)=\left[-\frac{2}{3} z(1-z) \frac{\partial}{\partial z}+(2 z-1)\right] \gamma, \\
& \beta(\gamma)=\alpha(\gamma)-\gamma .
\end{aligned}
$$

The function $\gamma$ is

$$
\gamma=-\frac{3 \Gamma\left(\frac{5}{2}+\nu\right) \Gamma\left(\frac{5}{2}-\nu\right)}{64 \pi^{2} a^{4} m^{2}} F\left(\frac{5}{2}+\nu, \frac{5}{2}-\nu ; 3 ; z\right),
$$

where $\nu=\sqrt{\frac{1}{4}-a^{2} m^{2}}$.

To evaluate $\bar{G}_{\text {spin-1 }}^{a c^{\prime}}(-2 \Lambda, z)$ we note that near $m^{2}=-2 \Lambda$ we can expand $G_{\text {spin-1 }}^{a c^{\prime}}$ in powers of $\varepsilon=m^{2}+2 \Lambda$. Thus for small $\varepsilon$ one has

$$
G_{\mathrm{spin}-1}^{a c^{\prime}}(\varepsilon-2 \Lambda, z)=A^{a c^{\prime}} \varepsilon^{-1}+B^{a c^{\prime}}+C^{a c^{\prime}} \varepsilon+\mathrm{O}\left(\varepsilon^{2}\right)
$$

Here $A^{a c^{\prime}}$ is given by

$$
A^{a c^{\prime}}=\frac{15}{16} \pi^{-2} a^{-4}\left[(2 z-1) g^{a c^{\prime}}+(2 z-2) n^{a} n^{c^{\prime}}\right] .
$$

Now since $Q_{0}^{a c^{\prime}}$ has the same singularity at $\varepsilon=0$,

$$
Q_{0}^{a c^{\prime}}=A^{a c^{\prime}} \varepsilon^{-1},
$$

their difference is regular near $\varepsilon=0$. Hence for $m^{2}=-2 \Lambda+\varepsilon$ one has

$$
G_{\mathrm{spin}-1}^{a c^{\prime}}(-2 \Lambda+\varepsilon, z)-Q_{0}^{a c^{\prime}}(-2 \Lambda+\varepsilon, z)=B^{a c^{\prime}}+C^{a c^{\prime}} \varepsilon+\mathrm{O}\left(\varepsilon^{2}\right) \text {. }
$$

To find $\bar{G}_{\text {spin-1 }}^{a c^{\prime}}$ one takes the derivative of the previous equation with respect to $-m^{2}$ to obtain $\bar{G}_{\text {spin- } 1}^{a c^{\prime}}(-2 \Lambda, z)=-C^{a c^{\prime}}$.

Now to evaluate $C^{a c^{\prime}}$, one expands $\gamma$ in powers of $\varepsilon$ and takes the linear term. Since near $\varepsilon=0$

$$
\gamma(z)=\gamma_{-1}(z) \varepsilon^{-1}+\gamma_{0}(z)+\gamma_{1}(z) \varepsilon+O\left(\varepsilon^{2}\right),
$$

one finds

$$
\bar{G}_{\mathrm{spin}-1}^{a c^{\prime}}=-\alpha\left(\gamma_{1}\right) \mathrm{g}^{a c^{\prime}}-\beta\left(\gamma_{1}\right) n^{a} n^{c^{\prime}}
$$

where

$$
\begin{aligned}
\gamma_{1}(z)=\frac{3}{80 a^{2} \pi^{2}} & {\left[\left(\log (1-z) \log (z)+\operatorname{li}_{2}(1-z)\right)+\frac{9}{10 z}-\frac{3}{4(z-1)^{2}}\right.} \\
& \left.-\frac{10350 \pi^{2}-4041}{500}+\log (1-z)\left[\frac{27}{5 z}+\frac{9}{10 z^{2}}-\frac{99}{5}\right]\right]
\end{aligned}
$$


where the logarithmic integral function is

$$
\mathrm{li}_{2}(y)=-\int_{0}^{y} \frac{\log (\zeta)}{\zeta} \mathrm{d} \zeta
$$

Thus from (5.5), (5.13) and (5.15) we have the transverse part of the graviton propagator given by

$$
\begin{aligned}
& g_{\mathrm{T}}^{1}=\kappa\left[\left[\frac{-9}{10 z}-\frac{3}{5 z^{2}}+\frac{9}{2(z-1)}+\frac{66}{5}\right]+\log (1-z)\left[-\frac{3}{5 z^{2}}-\frac{3}{5 z^{3}}+\frac{36}{5}\right]\right] \\
& g_{\mathrm{T}}^{2}=\kappa\left[\left[\frac{216 z-456}{5}-\frac{36}{5 z}-\frac{24}{5 z^{2}}+\frac{36}{z-1}\right]+\log (1-z)\left[\frac{24}{z}-\frac{24}{5 z^{2}}-\frac{24}{5 z^{3}}-\frac{72}{5}\right]\right], \\
& g_{\mathrm{T}}^{3}=\kappa\left[\left[-\frac{9}{10 z}-\frac{3}{5 z^{2}}-\frac{9}{2(z-1)}-\frac{117}{5}\right]+\log (1-z)\left[-\frac{3}{5 z^{2}}-\frac{3}{5 z^{3}}-\frac{54}{5}\right]\right], \\
& g_{\mathrm{T}}^{4}=\kappa\left[\left[\frac{27}{5 z}+\frac{18}{5 z^{2}}-\frac{9}{z-1}-6\right]+\log (1-z)\left[\frac{18}{5 z^{2}}+\frac{18}{5 z^{3}}-\frac{36}{5}\right]\right] \\
& g_{\mathrm{T}}^{5}=\kappa\left[\left[\frac{54 z-144}{5}+\frac{18}{5 z}+\frac{12}{5 z^{2}}\right]+\log (1-z)\left[\frac{6}{z}+\frac{12}{5 z^{2}}+\frac{12}{5 z^{3}}-\frac{54}{5}\right]\right],
\end{aligned}
$$

where $\kappa=1 / 288 \pi^{2} a^{2}$.

\section{The transverse traceless part of the propagator}

We evaluate $\boldsymbol{G}_{\mathrm{TT}}$, the transverse-traceless part of the propagator, using the methods of [6]. We know that it can be expressed in the form

$$
\boldsymbol{G}_{\mathrm{TT}}=\alpha(\mu) \boldsymbol{O}_{1}+\beta(\mu) \boldsymbol{O}_{2}+\tau(\mu) \boldsymbol{O}_{3}+\delta(\mu) \boldsymbol{O}_{4}+\varepsilon(\mu) \boldsymbol{O}_{5} .
$$

We will use the equation of motion for $\boldsymbol{G}_{\mathrm{TT}}$, and the tracelessness and transverseness conditions, to solve for the undetermined coëfficient functions $\{\alpha, \beta, \tau, \delta, \varepsilon\}$.

\subsection{TRACELESSNESS}

We require that $G_{\mathrm{TT}}^{a b c^{\prime} d^{\prime}}$ be traceless on both sets of indices - primed and unprimed. Tracing on the primed or unprimed indices, one finds that tracelessness requires

$$
(4 \alpha+2 \tau+\delta) g^{a b}+(\beta+4 \delta-4 \varepsilon) n^{a} n^{b}=0
$$


and hence that

$$
\begin{aligned}
& 4 \alpha+2 \tau+\delta=0 \\
& \beta+4 \delta-4 \varepsilon=0
\end{aligned}
$$

Thus there are three independent coefficient functions. In fact, one could define three traceless bitensor objects, in terms of which any traceless terms in the propagator can be written. If one calls the three traceless objects $\left\{\boldsymbol{T}_{1}, \boldsymbol{T}_{2}, \boldsymbol{T}_{3}\right\}$ and writes $\boldsymbol{G}_{\mathrm{TT}}$ as

$$
\boldsymbol{G}_{\mathrm{TT}}=X(\mu) \boldsymbol{T}_{1}+Y(\mu) \boldsymbol{T}_{2}+Z(\mu) \boldsymbol{T}_{3},
$$

then the conditions of $(6.3 \mathrm{a}, \mathrm{b})$ are automatically satisfied. We will employ one such set later in this section.

6.2. TRANSVERSENESS

$\boldsymbol{G}_{\mathrm{TT}}$ is transverse; that is, it obeys

$$
\nabla_{a} G_{\mathrm{TT}}^{a b c^{\prime} d^{\prime}}=0
$$

(where one could have taken the divergence on any one of the indices of $\boldsymbol{G}_{\mathrm{TT}}$ ). Substituting in the form of $\boldsymbol{G}_{\mathrm{TT}}$ (6.1) into (6.5), one obtains the equation

$$
c_{1}(\mu) n^{b} g^{c^{\prime} d^{\prime}}+c_{2}(\mu) n^{b} n^{c^{\prime}} n^{d^{\prime}}+2 c_{3}(\mu) n^{\left(c^{\prime}\right.} g^{\left.d^{\prime}\right) b}=0,
$$

where the three vanishing coefficient functions are

$$
\begin{aligned}
& c_{1}(\mu)=\alpha^{\prime}-2(A+C) \tau+\left(\delta^{\prime}+3 A \delta\right)+2 C \varepsilon=0, \\
& c_{2}(\mu)=\beta^{\prime}+3 A \beta+\delta^{\prime}+2 C \delta-2\left(\varepsilon^{\prime}+2(A+2 C) \varepsilon\right)=0, \\
& c_{3}(\mu)=-\left(\tau^{\prime}+4(A+C) \tau\right)+C \delta+\left(\varepsilon^{\prime}+4 A \varepsilon\right)=0,
\end{aligned}
$$

and ' denotes $d / d \mu$. Only two of these three equations are independent in our case, because any two of them, together with the tracelessness conditions $(6.3 \mathrm{a}, \mathrm{b})$ imply the third.

\subsection{THE EQUATION OF MOTION}

From the definition of the transverse-traceless mode functions $\left\{\boldsymbol{h}_{\mathrm{TT} n}^{i}\right\}$, and the definition of the transverse-traceless part of the propagator (2.22), one can see that $\boldsymbol{G}_{\mathrm{TT}}$ obeys the equation of motion

$$
\left(-\square+\frac{2}{3} \Lambda\right) G_{\mathrm{TT}}^{a b c^{\prime} d^{\prime}}=\sum_{n=0}^{\infty} h_{\mathrm{TT} n}^{a b}(x) h_{\mathrm{TT} n}^{c^{\prime d^{\prime}}}\left(x^{\prime}\right) .
$$


In the future, we will write the scalar operator appearing in (6.8) as

$$
(-\square+2)
$$

where we've used $\Lambda=3 a^{-2}$ and restricted ourselves to the unit sphere $a=1$. We will restore $a$ at the end. The source-term appearing on the right-hand side of (6.8) is not a delta function. It is evaluated in terms of elementary functions of the geodesic separation in appendix B, using methods developed in previous sections. We find

$$
\sum_{n=0}^{\infty} h_{\mathrm{TT} n}^{a b}(x) h_{\mathrm{TT} n}^{c^{\prime} d^{\prime}}\left(x^{\prime}\right)=\Theta^{a b c^{\prime} d^{\prime}}(z)
$$

with $\Theta$ given by

$$
\begin{aligned}
& \vartheta^{1}=3 \kappa \frac{-12 z^{2}+20 z-5}{(1-z)^{2}}, \\
& \vartheta^{2}=3 \kappa \frac{8\left(3 z^{2}-10 z+10\right)}{(1-z)^{2}}, \\
& \vartheta^{3}=3 \kappa \frac{2\left(9 z^{2}-15 z+5\right)}{(1-z)^{2}}, \\
& \vartheta^{4}=3 \kappa \frac{4 z(3 z-5)}{(1-z)^{2}}, \\
& \vartheta^{5}=3 \kappa \frac{2\left(9 z^{2}-20 z+10\right)}{(1-z)^{2}},
\end{aligned}
$$

and where $\kappa=1 / 288 \pi^{2}$ as in the previous sections.

\subsection{SOLUTION OF THE EQUATION OF MOTION}

One can now find the equation of motion for the coefficient functions introduced earlier by evaluating the components of the equation

$$
(\square-2) G_{\mathrm{TT}}^{a b c^{\prime} d^{\prime}}=-\Theta^{a b c^{\prime} d^{\prime}}(z)
$$

The evaluation of $\square \boldsymbol{G}_{\mathrm{TT}}$ is simplified because for $\boldsymbol{O}_{i}$ any of our basis objects, and $f(\mu)$ any well-behaved function of $\mu$, one has

$$
\square\left(f \boldsymbol{O}_{i}\right)=(\square f) \boldsymbol{O}_{1}+(f)\left(\square \boldsymbol{O}_{i}\right)
$$


One can easily express $\square f$ as a function of $z$ or $\mu$ :

$$
\begin{aligned}
\square f & =\left[\frac{\mathrm{d}^{2}}{\mathrm{~d} \mu^{2}}+3 A \frac{\mathrm{d}}{\mathrm{d} \mu}\right] f \\
& =\left[z(1-z) \frac{\mathrm{d}^{2}}{\mathrm{~d} z^{2}}+2(1-2 z) \frac{\mathrm{d}}{\mathrm{d} z}\right] f .
\end{aligned}
$$

The expressions for $\square O_{i}$ can be found in appendix $A$. Using them, one finds $(\square-2) \boldsymbol{G}_{\mathrm{TT}}=\boldsymbol{K}$, with

$$
\begin{aligned}
& k_{1}=(\square-2) \alpha+2\left(C^{2}+A^{2}\right) \delta, \\
& k_{2}=(\square-2) \beta-8\left(C^{2}-A C+A^{2}\right) \beta+8(C+A)^{2} \varepsilon, \\
& k_{3}=(\square-2) \tau-2(C+A)^{2} \tau+4 A C \varepsilon, \\
& k_{4}=(\square-2) \delta-4\left(A^{2}+C^{2}\right) \delta+\left(A^{2}+C^{2}\right) \beta+4(A+C)^{2} \tau-4(A+C)^{2} \varepsilon, \\
& k_{5}=(\square-2) \varepsilon-2\left(3 C^{2}+2 A C+3 A^{2}\right) \varepsilon+2 A C \beta+4(C+A)^{2} \tau, \\
& k_{6}=-2\left(C^{2}-A^{2}\right)(-4 \varepsilon+4 \delta+\beta),
\end{aligned}
$$

where $A(\mu)$ and $C(\mu)$ are the bitensor manipulation functions defined in table 1 . Note that there is an $O_{6}$ term for general coefficients $\{\alpha, \beta, \tau, \delta, \varepsilon\}$. However in our case, because of the tracelessness condition (6.3b), the $k_{6}$ term is identically zero. The five objects $O_{1}, \ldots, O_{5}$ are linearly independent. Thus the equation of motion (6.10) implies that $k_{i}+\vartheta_{i}=0$ for $i=1, \ldots, 5$. Because of the tracelessness conditions of $(6.3 \mathrm{a}, \mathrm{b})$, only three of these equations are independent. Since $\boldsymbol{O}_{1}$ and $\boldsymbol{O}_{4}$ are pure trace, the five equations are thus linear combinations of the equations

$$
\begin{aligned}
& k_{2}+\vartheta_{2}=0, \\
& k_{3}+\vartheta_{3}=0, \\
& k_{5}+\vartheta_{5}=0 .
\end{aligned}
$$

We now express $\boldsymbol{G}_{\mathrm{TT}}$ in a traceless basis

$$
G_{\mathrm{TT}}=X(\mu) T_{1}+Y(\mu) T_{2}+Z(\mu) T_{3}
$$


where the traceless objects are

$$
\begin{aligned}
& T_{1}=\frac{1}{16} O_{1}+O_{2}-\frac{1}{4} O_{4}, \\
& T_{2}=O_{3}-\frac{1}{2} O_{1}, \\
& T_{3}=O_{5}+4 O_{2} .
\end{aligned}
$$

From (6.1), the coefficient functions are

$$
\begin{aligned}
& \alpha=\frac{1}{16}(X-8 Y), \\
& \beta=X+4 Z \\
& \tau=Y \\
& \delta=-\frac{1}{4} X \\
& \varepsilon=Z
\end{aligned}
$$

In terms of these new variables, the two transverse equations (6.7) are

$$
\begin{array}{r}
32 C Z-8\left(Y^{\prime}+4(A+C) Y\right)-3\left(X^{\prime}+4 A X\right)=0 \\
4\left(Y^{\prime}-4(C+A) Y\right)-C X=0
\end{array}
$$

and the three wave equations (6.16) are

$$
\begin{gathered}
4\left(Z^{\prime \prime}+3 A Z^{\prime}-2\left(1+3(A-C)^{2}\right) Z\right) \\
+\left(X^{\prime \prime}+3 A X^{\prime}-2\left(1+4\left(C^{2}-A C+A^{2}\right)\right) X\right)=-\vartheta_{2} \\
\left(Y^{\prime \prime}+3 A Y^{\prime}-2\left(1+(A+C)^{2}\right) Y\right)+4 A C Z=-\vartheta_{3} \\
\left(Z^{\prime \prime}+3 A Z^{\prime}-2\left(1+3 C^{2}-2 A C+3 A^{2}\right) Z\right) \\
+4(C+A)^{2} Y+2 A C X=-\vartheta_{5} .
\end{gathered}
$$

Introducing the functions $W(\mu)$ and $U(\mu)$ defined by

$$
\begin{gathered}
W=X+\frac{8}{3} Y, \\
U=Y-Z,
\end{gathered}
$$


one can decouple the three equations (6.18), obtaining:

$$
\begin{aligned}
W^{\prime \prime}+7 A W^{\prime}-10 W & =-\vartheta_{2}-\frac{8}{3} \vartheta_{3}+4 \vartheta_{5}, \\
U & =-\frac{3}{32 C}\left(W^{\prime}+8 A W\right), \\
Y & =-\frac{3}{40}\left(W+\frac{4}{C} U^{\prime}+\frac{16 A}{C} U\right) .
\end{aligned}
$$

In terms of $z=\cos ^{2}\left(\frac{1}{2} \mu\right)$ one can rewrite (6.21)-(6.23) as

$$
\begin{gathered}
z(1-z) \frac{\mathrm{d}^{2} W}{\mathrm{~d} z^{2}}+4(1-2 z) \frac{\mathrm{d} W}{\mathrm{~d} z}-10 W=-\frac{5}{18 \pi^{2}} \frac{1}{(1-z)^{2}}, \\
U=\frac{3}{16}\left(z(z-1) \frac{\mathrm{d} W}{\mathrm{~d} z}+2(2 z-1) W\right), \\
Y=\frac{3}{40}\left(8 z(z-1) \frac{\mathrm{d} U}{\mathrm{~d} z}+16(2 z-1) U-W\right) .
\end{gathered}
$$

Once $W$ is determined from (6.24), $U$ can be obtained from (6.25), and then $Y$ from (6.26). This completely determines the functions $\{X, Y, Z\}$, and thus the functions $\{\alpha, \beta, \gamma, \delta, \varepsilon\}$, in terms of $W$.

Eq. (6.24) is a hypergeometric equation for $W$, with an inhomogeneous source term. The general solution is of the form

$$
W(z)=W_{\mathrm{p}}(z)+\frac{r}{\pi^{2}} W_{1}(z)+s W_{2}(z),
$$

where $W_{1}$ and $W_{2}$ are linearly independent solutions of the homogeneous hypergeometric equation with parameters $a=5, b=2$, and $c=4$. The function $W_{\mathrm{p}}$ is any particular solution to the inhomogeneous equation, and $r$ and $s$ are constants. The homogeneous solutions can be taken as [16]

$$
\begin{aligned}
& W_{1}=F(5,2 ; 4 ; z)=\frac{2-z}{2(1-z)^{3}}, \\
& W_{2}=F(5,2 ; 4 ; 1-z)=\frac{1+z}{2 z^{3}} .
\end{aligned}
$$

One can easily verify that a particular solution to the inhomogeneous equation for $W_{\mathrm{p}}$ is

$$
W_{\mathrm{p}}(z)=\frac{5}{54 \pi^{2}}\left(\frac{1}{z^{2}}+\frac{1}{z^{3}}\right) \log (1-z)+\frac{5}{36 \pi^{2}}\left(\frac{1}{z}+\frac{2}{3 z^{2}}+\frac{1}{1-z}\right) .
$$


Because we are considering the euclidean vacuum state in de Sitter space, the solution $W$ must be regular at $z=0$ [7]. Because $W_{\mathrm{p}}$ and $W_{1}$ are regular at $z=0$, and $W_{2}$ is singular at $z=0$, this condition forces $s$ to vanish. Thus

$$
W(z)=W_{\mathrm{p}}(z)+\frac{r}{\pi^{2}} W_{1} .
$$

Rescaling to the general radius 4-sphere, we obtain the following general solution to the equations for the transverse-traceless part of the propagator:

$$
\begin{aligned}
& g_{\mathrm{TT}}^{1}=\alpha(z)=\kappa\left[\frac{3}{2 z}+\frac{\log (1-z)+1}{z^{2}}+\frac{\log (1-z)}{z^{3}}-\frac{3}{2(z-1)}+\frac{27 r}{5(z-1)^{2}}\right. \\
& \left.-\frac{27 r}{5(z-1)^{3}}-\frac{60 \log (1-z)+324 r+20}{5}\right] \text {, } \\
& g_{\mathrm{TT}}^{2}=\beta(z)=\kappa\left[\frac{12-40 \log (1-z)}{z}+\frac{8 \log (1-z)+8}{z^{2}}+\frac{8 \log (1-z)}{z^{3}}+\frac{-216 r-12}{z-1}\right. \\
& \left.+\frac{1296 r}{5(z-1)^{2}}-\frac{1296 r}{5(z-1)^{3}}+\frac{120 \log (1-z)+648 r+40}{5}\right] \\
& g_{\mathrm{TT}}^{3}=\tau(z)=\kappa\left[\frac{3}{2 z}+\frac{\log (1-z)+1}{z^{2}}+\frac{\log (1-z)}{z^{3}}-\frac{3}{2(z-1)}+\frac{27 r}{5(z-1)^{2}}\right. \\
& \left.+\frac{27 r}{5(z-1)^{3}}+\frac{90 \log (1-z)+486 r+30}{5}\right] \text {, } \\
& g_{\mathrm{TT}}^{4}=\delta(z)=\kappa\left[-9 / z+\frac{-6 \log (1-z)-6}{z^{2}}-\frac{6 \log (1-z)}{z^{3}}+\frac{9}{z-1}-\frac{162 r}{5(z-1)^{2}}\right. \\
& \left.+\frac{162 r}{5(z-1)^{3}}+\frac{60 \log (1-z)+324 r+20}{5}\right] \text {, } \\
& g_{\mathrm{TT}}^{5}=\varepsilon(z)=\kappa\left[\frac{-10 \log (1-z)-6}{z}+\frac{-4 \log (1-z)-4}{z^{2}}\right. \\
& -\frac{4 \log (1-z)}{z^{3}}+\frac{6-54 r}{z-1} \\
& \left.+\frac{162 r}{5(z-1)^{2}}-\frac{162 r}{5(z-1)^{3}}+\frac{90 \log (1-z)+486 r+30}{5}\right] \text {. }
\end{aligned}
$$

The value of the constant $r$ appearing in (6.32) will be obtained by examination of the behavior of the full propagator in the short distance limit $\mu \rightarrow 0$. 


\section{The full graviton propagator}

\subsection{BEHAVIOR AS $(\mu / a) \rightarrow 0$}

We now have all the terms necessary for the evaluation of the graviton propagator. However, when transverse-traceless part of the propagator was evaluated in sect. 6, we were left with an undetermined constant $r$. The value of this constant is determined by requiring that graviton propagator match the standard flat-space propagator in the short distance limit $\mu / a \rightarrow 0$.

In flat space, the graviton propagator for our choice of gauge-fixing term can be written as $[13,15]$

$$
\boldsymbol{D}=(1 / 2 \rho)\left[\boldsymbol{O}_{3}-\boldsymbol{O}_{1}\right] D\left(x, x^{\prime}\right)
$$

where $\rho=1 / 32 \pi \mathscr{G}$ as before, and $D\left(x, x^{\prime}\right)$ is the massless scalar two-point function. The scalar propagator in flat space is of the form [6]

$$
D\left(x, x^{\prime}\right)=\frac{1}{4 \pi^{2} \mu^{2}}
$$

in four dimensions, and since $(1-z) \rightarrow\left(\mu^{2} / 4 a^{2}\right)$ as $(\mu / a) \rightarrow 0$, one has

$$
D=\lim _{a \rightarrow \infty} \frac{1}{32 \pi^{2} a^{2}}(1-z)^{-1}\left[O_{3}-O_{1}\right]
$$

This means that the $\boldsymbol{O}_{1}$ and $\boldsymbol{O}_{3}$ terms of the full propagator must be proportional to $(1-z)^{-1}$ as $(\mu / a) \rightarrow 0$. The other terms must then either be finite or go to infinity more slowly than $(1-z)^{-1}$ as $z \rightarrow 1$. One therefore needs an approximate form for $G(-2 \Lambda, z)$ near $z=1$ to evaluate the propagator in the flat-space limit. With the aid of formulae for $F(a, b ; c ; 1-z)[16,17]$, one finds

$$
\begin{array}{r}
G(-2 \Lambda, z) \approx \frac{1}{16 \pi^{2} a^{2}}\left[-24\left(\log (1-z) \log (z)+\operatorname{Li}_{2}(1-z)\right)\right. \\
\left.+\frac{1}{1-z}-8 \log (1-z)-8\right]
\end{array}
$$

for $z \approx 1$. Here, $\mathrm{Li}_{2}(y)$ is the logarithmic integral function used in sect. 5. Note that one keeps all the terms in this expansion because our expression for the propagator involves its first derivative with respect to $z$. Using this limiting expression, we examine the $O_{1}$ and $O_{2}$ components of the full propagator, assembled from the 
various pieces we have found. As $\mu / a \rightarrow 0$, one finds

$$
\begin{aligned}
& \rho g^{1} \rightarrow r\left[\frac{3 a^{2}}{10 \pi^{2} \mu^{4}}+\frac{6 a^{4}}{5 \pi^{2} \mu^{6}}\right]-\frac{1}{8 \pi^{2} \mu^{2}}, \\
& \rho g^{2} \rightarrow r\left[\frac{3}{\pi^{2} \mu^{2}}+\frac{72 a^{2}}{5 \pi^{2} \mu^{4}}+\frac{288 a^{4}}{5 \pi^{2} \mu^{6}}\right] .
\end{aligned}
$$

Thus $r$ must vanish. It is only for $r=0$ that the curved space propagator has the correct short distance $(\mu / a) \rightarrow 0$ behavior. To the lowest order in $(1-z)$, one has

$$
\begin{aligned}
& \rho g^{1}=\frac{1}{32 \pi^{2} a^{2}(z-1)}-\frac{20 \log (1-z)-111}{1440 \pi^{2} a^{2}}+\mathrm{O}((1-z) \log (1-z)), \\
& \rho g^{2}=-\frac{60 \log (1-z)+16}{9 \pi^{2} a^{2}}+\mathrm{O}((1-z) \log (1-z)), \\
& \rho g^{3}=-\frac{1}{32 \pi^{2} a^{2}(z-1)}-\frac{100 \log (1-z)+11}{720 \pi^{2} a^{2}}+\mathrm{O}((1-z) \log (1-z)), \\
& \rho g^{4}=\frac{15 \log (1-z)+4}{18 \pi^{2} a^{2}}+\mathrm{O}((1-z) \log (1-z)), \\
& \rho g^{5}=-\frac{15 \log (1-z)+4}{18 \pi^{2} a^{2}}+\mathrm{O}((1-z) \log (1-z)) .
\end{aligned}
$$

The subdominant logarithmic terms do not show up in the flat space limit $a \rightarrow \infty$.

\subsection{THE COMPLETE GRAVITON PROPAGATOR}

With the constant $r$ determined to be 0 , one can now give a complete expression for the graviton propagator in de Sitter space. Adding (3.8), (4.10), (4.14), (5.26) and (6.32), one finds

$$
\begin{aligned}
G^{a b c^{\prime} d^{\prime}}= & g^{1}\left(g^{a b} g^{c^{\prime} d^{\prime}}\right)+g^{2}\left(n^{a} n^{b} n^{c^{\prime}} n^{d^{\prime}}\right)+g^{3}\left(g^{a c^{\prime}} g^{b d^{\prime}}+g^{a d^{\prime}} g^{b c^{\prime}}\right) \\
& +g^{4}\left(g^{a b} n^{c^{\prime}} n^{d^{\prime}}+n^{a} n^{b} g^{c^{\prime} d^{\prime}}\right)+g^{5}\left(4 n^{(a} g^{b)\left(c^{\prime}\right.} n^{\left.d^{\prime}\right)}\right)
\end{aligned}
$$


for

$$
\begin{aligned}
& g^{1}=\mathscr{K}\left[\frac{3}{5 z}+\frac{2}{5 z^{2}}-\frac{3}{z-1}+\frac{2}{(z-1)^{2}}+\frac{1}{(z-1)^{3}}+\frac{46}{5}\right. \\
& \left.+\log (1-z)\left(\frac{2}{5 z^{2}}+\frac{2}{5 z^{3}}-\frac{24}{5}\right)\right]+\rho^{-1} g_{l_{2}}^{1}+(4 \rho)^{-1} f(z), \\
& g^{2}=\mathscr{K}\left[\frac{216 z-416}{5}+\frac{24}{5 z}+\frac{16}{5 z^{2}}+\frac{40}{z-1}-\frac{48}{(z-1)^{2}}+\frac{48}{(z-1)^{3}}\right. \\
& \left.+\log (1-z)\left(-\frac{16}{z}+\frac{16}{5 z^{2}}+\frac{16}{5 z^{3}}+\frac{48}{5}\right)\right]+\rho^{-1} g_{l_{2}}^{2}, \\
& g^{3}=\mathscr{K}\left[\frac{3}{5 z}+\frac{2}{5 z^{2}}+\frac{3}{z-1}-\frac{4}{(z-1)^{2}}+\frac{1}{(z-1)^{3}}-\frac{87}{5}\right. \\
& \left.+\log (1-z)\left(\frac{2}{5 z^{2}}+\frac{2}{5 z^{3}}+\frac{36}{5}\right)\right]+\rho^{-1} g_{l_{2}}^{3}, \\
& g^{4}=\mathscr{K}\left[-\frac{18}{5 z}-\frac{12}{5 z^{2}}+\frac{6}{z-1}-\frac{6}{(z-1)^{3}}-2\right. \\
& \left.+\log (1-z)\left(-\frac{12}{5 z^{2}}-\frac{12}{5 z^{3}}+\frac{24}{5}\right)\right]+\rho^{-1} g_{l_{2}}^{4} \\
& g^{5}=\mathscr{K}\left[\frac{54 z-114}{5}-\frac{12}{5 z}-\frac{8}{5 z^{2}}+\frac{16}{z-1}-\frac{12}{(z-1)^{2}}+\frac{6}{(z-1)^{3}}\right. \\
& \left.+\log (1-z)\left(-\frac{4}{z}-\frac{8}{5 z^{2}}-\frac{8}{5 z^{3}}+\frac{36}{5}\right)\right]+\rho^{-1} g_{l_{2}}^{5} .
\end{aligned}
$$

In this expression:

$\mathscr{G}$ is Newton's constant,

$$
\begin{aligned}
& \rho=(32 \pi \mathscr{G})^{-1}, \\
& \mathscr{X}=\frac{\mathscr{G}}{9 \pi a^{2}} .
\end{aligned}
$$


The functions $f(z)$ and $g_{l_{2}}^{i}(z): i \in\{1,2,3,4,5\}$ are defined in (3.6) and (4.14).

\subsection{ANTIPODAL BEHAVIOR}

We examine the behavior of our solution as $z \rightarrow 0$. On the 4-sphere, this corresponds to the points $x$ and $x^{\prime}$ being on opposite sides of the sphere. The propagator, and each of its four parts, is regular at $z=0$. This should be no surprise, as we've consistently chosen the vector and scalar propagators for the euclidean vacuum state of de Sitter space when evaluating the graviton propagator. The limiting form for $G(-2 \Lambda, z)$ is

$$
G(-2 \Lambda, z)=\frac{1-3 z+z^{2}+\frac{1}{3} z^{3}}{16 \pi^{2} a^{2}}+\mathrm{O}\left(z^{4}\right),
$$

and thus near $z=0$ one obtains

$$
\left[\rho g^{1}, \rho g^{2}, \rho g^{3}, \rho g^{4}, \rho g^{5}\right] \approx \frac{371}{8640 \pi^{2} a^{2}}[1-(135 / 371),-16,-2,0,-4]+O(z) \text {. }
$$

As promised, the two-point function is regular for $x$ and $x^{\prime}$ antipodally separated.

\subsection{LARGE $-z$ BEHAVIOR}

We now consider the behavior of the graviton propagator in the limit as $z \rightarrow-\infty$, corresponding to large spacelike separations. The scalar propagator $G(-2 \Lambda, z)$ is approximated for large $z$ by employing the relationships between hypergeometric functions of $z$ and $1 / z[16,17]$. One finds

$$
G(-2 \Lambda, z)=\frac{1}{16 \pi^{2} a^{2}}(-z)^{(\sqrt{33}-3) / 2}\left[1-\frac{1}{4}(\sqrt{33}-3) z^{-1}+\mathrm{O}\left(z^{-2}\right)\right] .
$$

The longitudinal and pure-trace contributions from $G(-2 \Lambda, z)$ dominate the behavior of the propagator in the limit $z \rightarrow-\infty$ :

$$
\begin{aligned}
& \rho g_{\text {dom }}^{1}=p(z)(-16 \sqrt{33}+80)+\mathrm{O}\left(z^{-1}\right), \\
& \left.\rho g_{\text {dom }}^{2}=p(z)(-256 \sqrt{33}+1472)+\mathrm{O}\left(z^{-1}\right)\right), \\
& \rho g_{\text {dom }}^{3}=O\left(z^{-1}\right), \\
& \rho g_{\text {dom }}^{4}=p(z)\left(64 \sqrt{33}-368+O\left(z^{-1}\right)\right), \\
& \rho g_{\text {dom }}^{5}=p(z)\left(\frac{(504-88 \sqrt{33})}{3 \sqrt{33} z}+O\left(z^{-2}\right)\right),
\end{aligned}
$$

for $p(z)=\left(1 / 768 \pi^{2} a^{2}\right)(-z)^{(\sqrt{33}-3) / 2}$. 
The dominant large $-z$ behavior of the propagator is given by the pure-trace piece of the propagator and $\rho G_{/ 2}(4.9,4.14)$. This can be written as a differential operator acting on $G(-2 \Lambda, z)$ :

$$
\rho G_{\mathrm{dom}}^{a b c^{\prime} d^{\prime}}=\left[\frac{1}{\Lambda^{2}} \nabla^{a} \nabla^{b} \nabla^{c^{\prime}} \nabla^{d^{\prime}}+\frac{1}{2 \Lambda}\left(g^{a b} \nabla^{c^{\prime}} \nabla^{d^{\prime}}+g^{c^{\prime} d^{\prime}} \nabla^{a} \nabla^{b}\right)\right] G(-2 \Lambda, z) .
$$

It is not yet clear if the large- $z$ behavior of the propagator poses any physical problems for the theory of gravitons in de Sitter space. The propagator is not gauge invariant, and one hopes that physical objects, formed by taking derivatives of the propagator, fall off at large distances.

\section{Conclusion}

In this paper, the symmetric two-point function for gravitons is evaluated in closed form in four-dimensional de Sitter space. Because the vacuum is the de Sitter-invariant euclidean vacuum state, and the gauge-fixing term is de Sitterinvariant, the two-point function is a maximally symmetric bitensor. In our notation it is expressed in coordinate-free language, in terms of manifestly de Sitter-invariant geometrical objects.

The gauge-fixing term was chosen with two points in mind. First, it does not itself break de Sitter invariance (as, for example, axial gauge in QED breaks Lorentz invariance). Second, it is a "good" gauge in that it completely fixes all of the gauge degrees of freedom [14]. For this reason the two-point function is finite for separated points. It does not contain the gauge-artifact infra-red divergence that is present for "bad" choices of gauge, for example the transverse-tracelesssynchronous gauge [21].

Certain terms in the two-point function, for example the transverse term, cannot contribute to tree-level scattering amplitudes. In this sense, these terms are pure gauge artifacts. These types of terms do not contribute to tree-level scattering amplitudes because they are pure divergences, and it is easy to show that such pure divergences make no contribution to any tree-level scattering amplitudes [14].

The symmetric two-point function contains a term which grows at large spacelike separations. This pure-trace term is not a gauge artifact in the sense discussed above, because it will contribute to certain tree-level scattering amplitudes. We do not yet understand if this growing pure-trace term is the symptom of some underlying physical instability, or if it is harmless. The rate of growth of this term depends upon the (unphysical) choice of a gauge-fixing term. For the choice that has been made in this paper, the growth is a power law in the distance variable $z$. For different choices of gauge it grows logarithmically [21]. However it seems unlikely that there exist any choices of gauge in which the propagator will fall off at large spacelike distances. 
What is the importance of this long-distance behavior for the standard inflationary model of the early universe? To begin with, it is known that the long-distance growth of the graviton propagator arises from the low-frequency modes [7,22-24]. At frequencies which are small compared to the horizon scale, the amplitude of these modes approaches a constant value. If our universe went through an early inflationary epoch, one consequence of this low-frequency behavior is that the present-day spectrum of gravitational wave noise would be scale-free [25-29]. The crucial question is this: Is the growth of the graviton propagator at long distances a symptom of some underlying physical instability or inconsistency in de Sitter space? There are of course two possibilities.

The first possibility is that the growing modes in the graviton propagator are harmless. One might hope that these modes are pure gauge-artifacts, but this is not true: the modes in question do contribute to observable gauge-independent quantities. The questions are, do they make only finite contributions, and do physical quantities such as the correlation function of the scalar curvature $\left\langle R(x) R\left(x^{\prime}\right)\right\rangle$. have reasonable behavior at large spacelike distances? There is accumulating evidence that this is indeed the case, although we know of no proof. The evidence includes calculations of the anisotropy of the cosmic background radiation induced by quantum fluctuations of the gravitational field during an inflationary de Sitter phase [28, 29]. The anisotropy at large scales is due to the low-frequency modes, and it is finite: it shows no pathological behavior. In a similar vein, the graviton propagator for a dust-dominated universe also grows at large spacelike separations [21]. Since our present-day universe is essentially dust-dominated, and seems to be quantum-mechanically stable, the growth of the graviton propagator at large distances is clearly not anathema. A final argument is based on the notion that symmetry-breaking induced by infra-red divergences (in this case, supposed breaking of de Sitter invariance) is usually accompanied by the introduction of an arbitrary mass-scale. Indeed the de Sitter space graviton propagator in transversetraceless-synchronous gauge contains an arbitrary infra-red-cutoff mass-scale. However it can be demonstrated that all scattering amplitudes are independent of this cutoff [21]. This suggests that the Hubble radius acts as a physical infra-red cut-off, and that the effects of the growing graviton propagator are not felt beyond this distance.

The second possibility is that the long distance growth of the graviton propagator leads to an instability of de Sitter space, which is accompanied by a spontaneous breakdown of de Sitter invariance. This interpretation has been advocated by Antoniadis and Mottola, who argue that the long-distance growth of the pure-trace and transverse-traceless terms in the propagator lead to quantum instability of the metric [30]. They have not conclusively demonstrated, however, that this is the case. What they show is that a small perturbation of the stress-energy tensor gives rise to a growing term in the metric. However this growing metric perturbation may be pure-gauge; in order to prove that it is an instability, it is necessary to show that it 
induces a growing perturbation in some physical gauge-invariant quantity such as the scalar curvature. If it is true that the long-distance behavior of the graviton propagator leads to an instability of de Sitter space, it would be fatal to the inflationary models of the early universe. A sufficiently long inflationary phase - typically 65 e-foldings - would be impossible to achieve because the de Sitter phase would only last for a few e-folding times. It has been shown that a de Sitter space instability of this sort could not give rise to a satisfactory inflationary model [31].

This work can be extended in several ways. First, by imposing different boundary conditions, one can use identical methods to find the graviton propagator in antide Sitter space, where the scalar curvature is negative. Second, one can carry out the calculation for $n$-dimensional de Sitter space, which will allow the use of dimensional regularization. Finally, one can investigate more general choices of gauge-fixing term. For example, it may be possible to simplify the result by working in Landau gauge.

We would like to thank Profs. L. Ford and J. Iliopoulos, and all of the others who have helped us with this paper. We are grateful for the support of the Tufts Department of Physics and Astronomy during the period of its preparation.

Many thanks to the Tufts Department of Computer Science for their assistance in the preparation of the original manuscript.

\section{Appendix A}

\section{USEFUL DEFINITIONS AND RESULTS}

A.1. Curvature identities. In a maximally symmetric space with curvature parameter $a^{2}$, the Riemann tensor is [5]:

$$
R^{a b c d}=\frac{1}{a^{2}}\left[g^{a c} g^{b d}-g^{a d} g^{b c}\right]
$$

Hence, for a four-sphere

$$
R^{a c}=\frac{3}{a^{2}} g^{a c}
$$

and

$$
R=\frac{12}{a^{2}} .
$$

Using the above identities and the Einstein equation, the cosmological constant $\Lambda$ is

$$
\Lambda=\frac{1}{4} R=3 a^{-2} \text {. }
$$


A.2. Bitensor manipulation. All the identities given here can be derived from the formulae of table 1 in sect. 2. Other helpful relations can be found in appendix $C$ of [6].

$$
\begin{aligned}
\square O_{1}= & 0, \\
\square O_{2}= & -\left(C^{2}-A^{2}\right) O_{6}+2 A C O_{5} \\
& +\left(C^{2}+A^{2}\right) O_{4}+8\left(A C-\left(A^{2}+C^{2}\right)\right) O_{2}, \\
\square O_{3}= & 4(C+A)^{2} O_{5}+4(C+A)^{2} O_{4}-2(C+A)^{2} O_{3}, \\
\square O_{4}= & -4\left(C^{2}-A^{2}\right) O_{6}-4\left(C^{2}+A^{2}\right) O_{4}+2\left(C^{2}+A^{2}\right) O_{1}, \\
\square O_{5}= & +4\left(C^{2}-A^{2}\right) O_{6}-\left(6\left(C^{2}+A^{2}\right)+4 A C\right) O_{5} \\
& -4(C+A)^{2} O_{4}+4 A C O_{3}+8(C+A)^{2} O_{2}, \\
\square O_{6}= & 4\left(C^{2}+A^{2}\right) O_{6}-2\left(A^{2}+C^{2}\right) O_{1}-4\left(C^{2}-A^{2}\right) O_{4} .
\end{aligned}
$$

For $G$ any function of $z$, we now give a formula for

$$
J^{a b c^{\prime} d^{\prime}}=a^{4}\left(\nabla^{a} \nabla^{b}-\frac{1}{4} g^{a b} \square\right)\left(\nabla^{c^{\prime}} \nabla^{d^{\prime}}-\frac{1}{4} g^{c^{\prime} d^{\prime}} \square^{\prime}\right) G
$$

The five coëfficient functions of $\boldsymbol{J}$ are given by:

$$
\begin{aligned}
& j^{1}=\frac{1}{16}\left[G_{(4)}(z-1)^{2} z^{2}-G_{(2)} \frac{1}{2}\left(-4 z^{2}+4 z+4\right)+2 G_{(3)}(z-1) z(2 z-1)\right], \\
& j^{2}=G_{(4)}(z-1)^{2} z^{2}+4 G_{(3)}(z-1)^{2} z+2 G_{(2)}(z-1)^{2} \\
& j^{3}=\frac{1}{4} G_{(2)} \\
& j^{4}=-\frac{1}{4}\left[G_{(4)}(z-1)^{2} z^{2}+2 G_{(3)}(z-1) z(2 z-1)+2 G_{(2)}(z-1) z\right] \\
& j^{5}=-\frac{1}{2}\left[G_{(3)}(z-1) z+G_{(2)}(z-1)\right]
\end{aligned}
$$

where $f_{(i)} \equiv \mathrm{d}^{i} f / \mathrm{d} z^{i}$.

We now give a formula for the components of

$$
K^{a b c^{\prime} d^{\prime}}=a^{4} \nabla^{a} \nabla^{b} \nabla^{c^{\prime}} \nabla^{d^{\prime}} G
$$


The component functions for $\boldsymbol{K}$ are given by:

$$
\begin{aligned}
& k^{1}=\frac{1}{4}(2 z-1)^{2} G_{(2)}+\frac{1}{2}(2 z-1) G_{(1)}, \\
& k^{2}=(z-1)^{2} z^{2} G_{(4)}+4(z-1)^{2} z G_{(3)}+2(z-1)^{2} G_{(2)}, \\
& k^{3}=\frac{1}{4} G_{(2)}, \\
& k^{4}=\frac{1}{2}(z-1) z(2 z-1) G_{(3)}+2(z-1) z G_{(2)}, \\
& k^{5}=-\frac{1}{2}(z-1) z G_{(3)}-\frac{1}{2}(z-1) G_{(2)} .
\end{aligned}
$$

For $\alpha$ and $\beta$ any two biscalars, and for

$$
P^{a b c^{\prime} d^{\prime}}=a^{2} \nabla^{\left(d^{\prime}\right.} \nabla^{(b}\left[\alpha g^{\left.a) c^{\prime}\right)}+\beta n^{a)} n^{\left.c^{\prime}\right)}\right]
$$

the components of $\boldsymbol{P}$ are given by

$$
\begin{aligned}
& p^{1}=\frac{2 z-1}{2 z} \alpha-\frac{(2 z-1)^{2}}{4 z(z-1)} \beta, \\
& p^{2}=-z(z-1) \beta_{(2)}+(z+1) \beta_{(1)}-\frac{z+1}{z-1} \beta, \\
& p^{3}=-\frac{1}{8 z(z-1)} \beta-\frac{1}{4 z} \alpha+\frac{1}{4} \alpha_{(1)}, \\
& p^{4}=\frac{2 z^{2}-2 z+1}{2(z-1) z} \beta-\frac{1}{2}(2 z-1) \beta_{(1)}+(z-1) \alpha_{(1)}-\frac{z-1}{z} \alpha, \\
& p^{5}=-\frac{4 z-1}{8(z-1) z} \beta+\frac{3}{8} \beta_{(1)}-\frac{1}{4} z(z-1) \alpha_{(2)}+\frac{1}{4} \alpha_{(1)}(z-1)-\frac{z-1}{4 z} \alpha .
\end{aligned}
$$

A.3. 4-Sphere eigenfunctions of $\square$ We employ the following eigenfunctions of $\square$ in the text $[14,19,21]$. Note that the index $n$ always ranges over the non-negative integers $0,1,2, \ldots$.

The scalar eigenfunctions are

$$
\varphi_{n}^{i}(x): \quad \square \varphi_{n}^{i}(x)=\lambda_{n}^{(0)} \varphi_{n}^{i}(x) .
$$

Their spectrum is given by

$$
\begin{aligned}
\lambda_{n}^{(0)} & =-n(n+3) a^{-2} \\
\text { for } i & =1, \ldots, \frac{1}{6}(n+1)(n+2)(2 n+3)
\end{aligned}
$$


The vector eigenfunctions are

$$
\xi_{n}^{i a}(x): \quad \square \xi_{n}^{i a}(x)=\lambda_{n}^{(1)} \xi_{n}^{i a}(x),
$$

which are transverse:

$$
\nabla_{a} \xi_{n}^{i a}(x)=0
$$

Their spectrum is given by

$$
\begin{aligned}
& \lambda_{n}^{(1)}=-\left(n^{2}+5 n+3\right) a^{-2} \\
& \text { for } i=1, \ldots, \frac{1}{2}(n+1)(n+4)(2 n+5)
\end{aligned}
$$

The symmetric spin-2 eigenfunctions are

$$
h_{\mathrm{TT} n}^{i a b}(x): \quad \square h_{\mathrm{T} T n}^{i a b}(x)=\lambda_{n}^{(2)} h_{\mathrm{TT} n}^{i a b}(x),
$$

which are transverse:

$$
\nabla_{a} h_{\mathrm{T} \mathbf{T} n}^{i a b}(x)=0
$$

They are also traceless:

$$
g_{a b} h_{\mathrm{TT} n}^{i a b}=0
$$

Their spectrum is given by

$$
\begin{aligned}
& \lambda_{n}^{(2)}=-\left(n^{2}+7 n+8\right) a^{-2} \\
& \text { for } i=1, \ldots, \frac{5}{6}(n+1)(n+6)(2 n+7) .
\end{aligned}
$$

A.4. Evaluation of the first two scalar mode-sum terms. We wish to obtain the first two terms appearing in the mode-sum representation of a massive scalar propagator on the 4-sphere. They can be written as

$$
\begin{aligned}
& \phi_{0}\left(m^{2}, \mu\right)=\frac{\varphi_{0}(x) \varphi_{0}\left(x^{\prime}\right)}{-\lambda_{0}^{(0)}+m^{2}}, \\
& \phi_{1}\left(m^{2}, \mu\right)=\sum_{i=1}^{5} \frac{\varphi_{1}^{i}(x) \varphi_{1}^{i}\left(x^{\prime}\right)}{-\lambda_{1}^{(0)}+m^{2}} .
\end{aligned}
$$

In both cases, we know that the terms are functions of the geodesic distance alone because all the degenerate eigenfunctions of $\square$ have been summed-over with equal weight. The 0-mode, $\varphi_{0}$, is constant and normalised (in the sense of sect. 2). Thus

$$
\phi_{0}\left(m^{2}, \mu\right)=\phi_{0}\left(m^{2}\right)=\frac{3}{8 \pi^{2} a^{4} m^{2}} .
$$


We know that the $5 \varphi_{1}^{i}$ are normalised, and also that they correspond to the cartesian coördinates of the surface of a 4-sphere embedded in $R^{5}$. Hence,

$$
\sum_{i=1}^{5} \varphi_{1}^{i}(x) \varphi_{1}^{i}\left(x^{\prime}\right) \propto x \cdot x^{\prime}=a^{2} \cos (\mu / a)=a^{2}(2 z-1) .
$$

We therefore obtain

$$
\phi_{1}\left(m^{2}, \mu(z)\right)=\frac{15(2 z-1)}{8 \pi^{2} a^{2}\left(4+a^{2} m^{2}\right)} .
$$

\section{Appendix B}

EVALUATION OF THE $\sum h h^{\prime}$ SOURCE-TERM

For separated points, the transverse traceless portion of the bitensor delta function appearing in (2.15) is

$$
\begin{aligned}
\Theta^{a b c^{\prime} d^{\prime}} & =\sum_{n=0}^{\infty} h_{\mathrm{TT} n}^{a b}(x) h_{\mathrm{TT} n}^{c^{\prime} d^{\prime}}\left(x^{\prime}\right) \\
& =-\sum_{n=1}^{\infty} V^{a b}(x) V^{c^{\prime} d^{\prime}}\left(x^{\prime}\right)-\sum_{n=2}^{\infty} W^{a b}(x) W^{c^{\prime} d^{\prime}}\left(x^{\prime}\right)
\end{aligned}
$$

One can evaluate the right-hand side of (B.1) using the techniques of sects. 4 and 5 .

The longitudinal mode-sum can be expanded in terms of the scalar eigenfunctions of $\square$, giving

$$
\Omega^{a b c^{\prime} d^{\prime}}=\sum_{n=2}^{\infty} W^{a b}(x) W^{c^{\prime} d^{\prime}}\left(x^{\prime}\right)=\overline{\nabla^{a} \nabla^{b}} \overline{\nabla^{c^{\prime}} \nabla^{d^{\prime}}} \boldsymbol{\sigma}\left(x, x^{\prime}\right)
$$

where $\overline{\nabla^{a} \nabla^{b}}=\nabla^{a} \nabla^{b}-\frac{1}{4} g^{a b} \square$ is the traceless derivative, and

$$
\sigma\left(x, x^{\prime}\right)=\frac{1}{\Lambda} \sum_{n=2}^{\infty} \varphi_{n}(x) \varphi_{n}\left(x^{\prime}\right)\left(\frac{1}{-\lambda_{n}^{(0)}-\frac{4}{3} \Lambda}-\frac{1}{-\lambda_{n}^{(0)}}\right) .
$$

Using the scalar function $\tilde{G}\left(k ; m^{2}, z\right)$ introduced in sect. 4 , one may write

$$
\sigma\left(x, x^{\prime}\right)=\sigma(z)=\frac{1}{3}\left[\tilde{G}\left(2 ;-\frac{4}{3} \Lambda, z\right)-\tilde{G}(2 ; 0, z)\right] .
$$

Referring back to sect. 4 one finds

$$
\sigma(z)=\frac{1}{8 \pi^{2} a^{2}}\left[(3-5 z) \log (1-z)-\frac{37}{10} z+\frac{29}{15}\right] .
$$


Because we want the traceless part of $\nabla^{a} \nabla^{b}$ (see (4.11)), only the logarithmic term will count in the end. One thus obtains the components of $\Omega$

$$
\begin{aligned}
& \omega^{1}=6 \kappa \frac{2}{z-1}, \\
& \omega^{2}=6 \kappa \frac{-8(z-4)}{(z-1)^{2}}, \\
& \omega^{3}=6 \kappa \frac{-(3 z-4)}{(z-1)^{2}}, \\
& \omega^{4}=6 \kappa \frac{-2 z}{(z-1)^{2}}, \\
& \omega^{5}=6 \kappa \frac{-4(z-2)}{(z-1)^{2}},
\end{aligned}
$$

where $\kappa=1 / 288 \pi^{2} a^{2}$.

To evaluate the sum over the transverse modes, one writes

$$
\begin{aligned}
\Psi^{a b c^{\prime} d^{\prime}} & =\sum_{n=1}^{\infty} V^{a b}(x) V^{c^{\prime} d^{\prime}}\left(x^{\prime}\right)=2 \sum_{n=1}^{\infty} \frac{\nabla^{\left(d^{\prime}\right.} \nabla^{(b} \xi_{n}^{a)} \xi_{n}^{\left.c^{\prime}\right)}}{-\lambda_{n}^{(1)}-\Lambda} \\
& =2 \nabla^{\left(d^{\prime}\right.} \nabla^{(b} \tilde{Q}^{\left.a) c^{\prime}\right)}(1 ;-2 \Lambda, z)
\end{aligned}
$$

where the function $\tilde{Q}^{a c^{\prime}}\left(k ; m^{2}, z\right)$ introduced in sect. 5 is an incomplete sum over the transverse modes. Using results of that section, one finds that the components of $\Psi$ are

$$
\begin{aligned}
& \psi^{1}=9 \kappa\left(4-\frac{1}{(z-1)^{2}}\right), \\
& \psi^{2}=9 \kappa\left(\frac{16}{z-1}-\frac{24}{(z-1)^{2}}-8\right), \\
& \psi^{3}=9 \kappa(-6) \\
& \psi^{4}=9 \kappa\left(\frac{4}{(z-1)^{2}}-4\right), \\
& \psi^{5}=9 \kappa\left(\frac{4}{z-1}-\frac{2}{(z-1)^{2}}-6\right) .
\end{aligned}
$$


Thus $\Theta$ has components

$$
\begin{aligned}
& \vartheta^{1}=3 \kappa \frac{-12 z^{2}+20 z-5}{(1-z)^{2}}, \\
& \vartheta^{2}=3 \kappa \frac{8\left(3 z^{2}-10 z+10\right)}{(1-z)^{2}}, \\
& \vartheta^{3}=3 \kappa \frac{2\left(9 z^{2}-15 z+5\right)}{(1-z)^{2}}, \\
& \vartheta^{4}=3 \kappa \frac{4 z(3 z-5)}{(1-z)^{2}}, \\
& \vartheta^{5}=3 \kappa \frac{2\left(9 z^{2}-20 z+10\right)}{(1-z)^{2}} .
\end{aligned}
$$

This result can also be obtained by computing $\left(\square-\frac{2}{3} \Lambda\right)\left(G_{T}+G_{L}\right)$.

\section{References}

[1] S.W. Hawking and G.F.W. Ellis, The large scale structure of space-time (Cambridge University Press, 1980)

[2] S.W. Hawking and G.W. Gibbons, Phys. Rev. D15 (1977) 2752;

R.H. Brandenburger, Phys. Rev. Lett. 129B (1983) 397

[3] I. Antoniadis, J. lliopolos and T.N. Tomaras, Phys. Rev. Lett. 56 (1986) 1319

[4] A. Guth, Phys. Rev. D23 (1981) 347;

R.H. Brandenburger, Rev. Mod. Phys. 57 (1985) 1-60;

G.W. Gibbons, S.W. Hawking, and S.T.C. Siklos (eds.), The very early universe (Cambridge University Press, 1983)

[5] N.D. Birrell and PP.C.W. Davies, Quantum fields in curved space (Cambridge University Press, 1982)

[6] B. Allen and T. Jacobson, Commun. Math. Phys. 103 (1986) 669-692

[7] B. Allen, Phys. Rev. D32 (1985) 3136

[8] S.J. Christensen and M.J. Duff, Nucl. Phys. B170 [FS1] (1980) 480

[9] W.G. Unruh, Phys. Rev. D14 (1976) 870

[10] D. Eardley and J. Traschen, Large scale anisotropy of the cosmic backround radiation in Friedmann universes, NSF-ITP-85-122 preprint, University of California at Santa Barbara (1985)

[11] N.P. Myhrvold, Phys. Lett. 132B (1983) 308, Phys. Rev. D28, 2439 (1983);

E. Mottola, Phys. Rev. D31 (1985) 754, Phys. Rev. D33 (1986) 1616

[12] C.W. Misner, K.S. Thorne and J.A. Wheeler: Gravitation (San Francisco: Freeman 1972)

[13] N.H. Barth and S.M. Christensen, Phys. Rev. D28 (1983) 1876

[14] B. Allen, Phys. Rev. D34 (1986) 3670

[15] G. 't Hooft and M. Veltman, Ann. Inst. Henri Poincaré, vol. 20, n¹ (1974) 69-94

[16] M. Abramowitz and I.A. Stegun: Handbook of mathematical functions, U.S. Government Printing Office (Washington D.C., 1972) 
[17] A. Erdelyi, Higher transcendental functions (Malabar: Krieger 1981)

[18] S. Weinberg, Gravitation and cosmology: Principles and applications of the theory of general relativity (Wiley, New York, 1972)

[19] G.W. Gibbons and M.J. Perry, Nucl. Phys. B146 (1978) 90

[20] A.H. Najmi and A.C. Ottewil, Phys. Rev. D30 (1984) 1733-1741

[21] B. Allen, Nucl. Phys. B287 (1987) 743

[22] T.S. Bunch and P.C.W. Davies, Proc. R. Soc. London A360 (1978) 117, J. Phys. A11 (1978) 1315

[23] L.H. Ford and L. Parker, Phys. Rev. D16 (1977) 1601

[24] B. Allen and A. Folacci, Phys. Rev. D (1987), to be published

[25] A. Starobinskii, JETP Lett. 30 (1979) 682, Phys. Lett. 117B (1982) 175

[26] V.A. Rubakov, M.V. Sazhin and A.V. Veryaskin, Phys. Lett. 115B (1982) 189

[27] R. Fabbri and M.D. Pollock, Phys. Lett. 125B (1983) 445

[28] L.F. Abbott and M.B. Wise, Phys. Lett. 135B (1984) 279, Nucl. Phys. B224 (1984) 541

[29] L.F. Abbott and R.K. Schaefer, Ap. J. 308 (1986) 546

[30] I. Antoniadis and E. Mottola, Graviton fluctuations in de Sitter space, CERN-TH 4605 report

[31] W. Hiscock, Phys. Lett. B166 (1986) 285 